(C) 2020 Elsevier. This manuscript version is made available under the CC-BY-NC-ND 4.0 license http://creativecommons.org/licenses/by-nc-nd/4.0/

\title{
Low-dissipation finite element strategy for low Mach number reacting flows
}

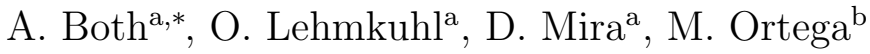 \\ ${ }^{a}$ Barcelona Supercomputing Center (BSC), Barcelona, Spain \\ ${ }^{b}$ Universitat Politècnica de Catalunya (UPC), ESEIAAT, Terrassa, Barcelona, Spain
}

\begin{abstract}
The present paper extends the conservative finite element convective scheme proposed by Charnyi et al.(Journal of Computational Physics 337, 2017, 289 - 308) originally formulated for incompressible flows to the low Mach regime. Similar to Lehmkuhl et al.(Journal of Computational Physics 390, 2019, 51 - 65) stabilisation is only introduced for the continuity equation by means of a non-incremental fractional-step method, modified in order to account for variable density flows. The final scheme preserves momentum and angular momentum for variable density flows. The error of kinetic energy conservation is of order $\mathcal{O}\left(\delta t h^{k+1}\right)$, thus dissipation is limited. Standard stabilised finite elements are used for the scalars. Time integration is carried out by means of an explicit third order Runge-Kutta scheme for all equations. The proposed strategy is tested on a set of relevant cases with available reference data using large-eddy simulations. First, an anisothermal turbulent channel flow is assessed. Later, a technically premixed turbulent flame in a swirl-stabilized configuration is considered. And finally, a turbulent jet
\end{abstract}

\footnotetext{
${ }^{*}$ Corresponding author

Email address: ambrus.both@bsc.es (A. Both)
} 
diffusion flame in a low-velocity co-flow has been studied. In all cases the performance of the presented low Mach formulation is fairly good, showing better accuracy than skew-symmetric like strategies.

Keywords: Low Mach, Finite element, Large-eddy simulation, Combustion, Low dissipation schemes

\section{Introduction}

2

Modelling of combustion problems of practical interest is a complex task as it involves the solution of chemically reactive flows interacting with turbulence. The multicomponent flow is characterised by high density ratios as a consequence of heat release and the kinetic energy has to be correctly dissipated by viscous forces [1]. This aspect motivates the seek for low-dissipation strategies that can be used to solve turbulent reactive flow calculations with high accuracy.

In practice, flame propagation is typically a slow process, thus the flow velocities are kept moderate to ensure flame stability, and to provide sufficient residence time for the reactions to complete. These processes are characterised by moderate to high Reynolds numbers, but usually take place at low Mach numbers, for instance stationary gas turbines or aero-engines operate at these conditions $[2,3]$. Thus, it is known, that turbulence has a strong effect on the flow, but compressibility can be negligible when the burners operate at these conditions. Therefore, low Mach number approximations of the Navier-Stokes equations are commonly used to model the flow on these applications, since they can be solved more efficiently from the numerical point of view. 
It has been demonstrated that large-eddy simulation (LES) is a powerful tool for solving problems of industrial interest, and it has been extensively applied in the numerical analysis of turbulent reacting flows at the low Mach number limit. LES addresses highly unsteady phenomena that characterises these turbulent problems. It has been successfully applied using different combustion models on various reactive cases $[1,2,4]$. A collective requirement for the numerical methods irrespective of the choice of combustion model, is the accurate prediction of the velocity field, since advection plays a major role in the transport of the reacting components.

One of the key aspects in achieving high-fidelity predictions of the velocity field, is the formulation of the discrete operator applied for the convective transport of momentum. This topic has been extensively studied in incompressible $[5,6,7]$ and compressible $[8,9,10,11,12]$ flows likewise. The different operators are characterised by whether they inherently adhere to the conservation of physical quantities like kinetic energy, or momentum. More specifically, in the case of finite elements, it is well established, that kinetic energy conservation can be ensured by devising the convective term of the momentum equation such, that it preserves skew-symmetry at the discrete level [13]. Charnyi et al. [5] introduced a discretization strategy for this term, that ensures the conservation of linear and angular momentum besides kinetic energy. Their method is developed for incompressible flows using mixed finite elements to stabilise pressure. Recently, this method has been extended to equal order finite elements by Lehmkuhl et al. [14] and it has been tested in fully turbulent incompressible flows with success. Recently Coppola et al. [12] classified a family of commonly used discretization 
strategies that stem from the splitting of the convective term. The present paper uses a different splitting method detailed below.

This study further extends the energy, momentum, and angular momentum conserving (EMAC) scheme to the low Mach regime. The underlying idea is to use a change of variables to preserve the fundamental properties of the incompressible implementation. In this case $\mathbb{A} \equiv \sqrt{\rho} \mathbf{u}$ is used in order to express the momentum flux as a vector product of two equal terms: $\rho \mathbf{u} \otimes \mathbf{u}=\mathbb{A} \otimes \mathbb{A}$. Such $\sqrt{\rho}$ formalism, appears in many studies related to variable density flows. Van Driest [15] introduced a scaling law for turbulent thermal boundary layers applying this transformation. Various studies used the formalism to study the effects of compressibility on forced and decaying turbulence in ideal gas $[16,17,18]$. More recently Rozema et al. [11] developed a conservative discretization scheme for compressible flows using finite volume method by using a $\sqrt{\rho}$ formalism in the state vector. In the present study we introduce a scheme, where the transport of $\mathbb{A}$ is not required.

Similar to Lehmkuhl et al. [14] the pressure stabilization is done with a non-incremental fractional-step method, however here, the approach of Nicoud [8] is used to incorporate variable density effects. Scalar equations are solved by means of stabilised finite elements to describe the thermochemical state of the fluid. The final set of equations is time integrated using an explicit third order Runge-Kutta method.

The next section introduces the low Mach equations in the weak form, followed by the derivation of the EMAC formulation for variable density flows. Then, we present a comprehensive analysis of the conservation properties of the EMAC operator in thermally coupled flows. In the results section, we test 
the developed scheme in representative cases of thermal flows with increased complexity: a fully developed channel flow with different temperature walls, a turbulent premixed swirling flame, and a non-premixed jet flame. Finally, conclusions are drawn.

\section{Low Mach number approximation}

In the low Mach number limit, the fully compressible Navier-Stokes equations can be simplified to decouple the thermochemical state of the flow from the momentum equation [19]. This splitting technique removes the acoustic modes forcing the flow to behave as incompressible, where the density can change due to temperature and composition variations. This can be achieved by a decomposition of the pressure into a spatially homogeneous thermodynamic pressure, and a hydrodynamic pressure: $P(x, y, z, t)=$ $P_{0}(t)+p(x, y, z, t)$

The thermodynamic pressure $P_{0}(t)$ is used in the equation of state, such as the ideal gas law:

$$
\rho=P_{0} \frac{W}{R_{u} T},
$$

where $W, R_{u}$ and $T$ represent the molecular weight, specific gas constant and temperature respectively. The hydrodynamic pressure $p(x, y, z, t)$ is used in the momentum equation and is determined by mass conservation. In closed domains, the thermodynamic pressure is time dependent such, that global mass conservation is ensured. In the case of the ideal gas law, the temporal evolution of thermodynamic pressure is calculated as:

$$
P_{0}(t)=\frac{M_{t_{0}} R_{u}}{\int_{\Omega} \frac{W}{T} \mathrm{~d} V}
$$


where $M_{t_{0}}=\int_{\Omega} \rho \mathrm{d} V$ is the initial mass in the domain. In open domains, the thermodynamic pressure is a known constant.

Considering a fluid flow in the low Mach number regime with variable composition, the problem consists of finding a velocity $\mathbf{u}$, a hydrodynamic pressure $p$, and scalars $\xi_{k}$ in domain $\Omega$ with boundary $\Gamma$ for the time interval $\left(t_{0}, t_{f}\right)$. In conservative form:

$$
\begin{aligned}
\partial_{t} \rho+\nabla \cdot(\rho \mathbf{u}) & =0 & & \text { on } \Omega \times\left(t_{0}, t_{f}\right), \\
\partial_{t}(\rho \mathbf{u})+\nabla \cdot(\rho \mathbf{u} \otimes \mathbf{u})+\nabla p-\nabla \cdot \boldsymbol{\tau}(\mathbf{u}) & =\mathbf{f} & & \text { on } \Omega \times\left(t_{0}, t_{f}\right), \\
\partial_{t}\left(\rho \xi_{k}\right)+\nabla \cdot\left(\rho \xi_{k} \mathbf{u}\right)+\nabla \cdot \boldsymbol{\Phi}_{k} & =S_{k} & & \text { on } \Omega \times\left(t_{0}, t_{f}\right),
\end{aligned}
$$

where $\boldsymbol{\tau}(\mathbf{u})=2 \mu \mathbf{S}^{\mathbf{D}}(\mathbf{u})$ is the viscous stress tensor with the deviatoric part of the strain rate: $\mathbf{S}^{\mathbf{D}}(\mathbf{u})=\frac{1}{2}\left(\nabla \mathbf{u}+\nabla^{\mathrm{T}} \mathbf{u}\right)-\frac{1}{3}(\nabla \cdot \mathbf{u}) \mathbf{I}, \mathbf{f}$ is the body force, and $\boldsymbol{\Phi}_{k}=-\rho D_{k} \nabla \xi_{k}$ and $S_{k}$ are the molecular flux and volumetric source term of scalar $k$ respectively. The system is closed using the equation of state (1). The temperature $T$, the molecular weight $W$, the dynamic viscosity $\mu$, and the diffusivity $D_{k}$ are known functions of the solved scalars $\xi_{k}$. The selection of transported scalars depends on the physics of the problem and will be introduced in the application cases. Note, that for the ideal gas law considered here, the density only depends on these scalars and the thermodynamic pressure.

The problem has to be appended with appropriate initial and boundary conditions. The initial conditions are:

$$
\begin{aligned}
& \mathbf{u}=\mathbf{u}_{0}, \quad \xi_{k}=\xi_{k, 0} \quad \text { on } \Omega \times\left\{t_{0}\right\} \\
& \text { and } \\
& P_{0}=P_{0}^{0} \quad \text { (for closed systems) } .
\end{aligned}
$$


Dirichlet and Neumann boundary conditions are defined for the velocity $\mathbf{u}$ on $\Gamma_{\mathbf{u}, D}$ and $\Gamma_{\mathbf{u}, N}$, and for the $k^{\text {th }}$ scalar $\xi_{k}$ on $\Gamma_{\xi_{k}, D}$ and $\Gamma_{\xi_{k}, N}$ respectively such, that $\Gamma_{\mathbf{u}, D} \cup \Gamma_{\mathbf{u}, N}=\Gamma$ and $\Gamma_{\xi_{k}, D} \cup \Gamma_{\xi_{k}, N}=\Gamma$,

$$
\begin{aligned}
\mathbf{u} & =\mathbf{u}_{D} & & \text { on } \Gamma_{\mathbf{u}, D} \times\left(t_{0}, t_{f}\right), \\
(-p \mathbf{I}+\boldsymbol{\tau}(\mathbf{u})) \cdot \mathbf{n} & =0 & & \text { on } \Gamma_{\mathbf{u}, N} \times\left(t_{0}, t_{f}\right), \\
\xi_{k} & =\xi_{k, D} & & \text { on } \Gamma_{\xi_{k}, D} \times\left(t_{0}, t_{f}\right), \\
\nabla \xi_{k} \cdot \mathbf{n} & =0 & & \text { on } \Gamma_{\xi_{k}, N} \times\left(t_{0}, t_{f}\right) .
\end{aligned}
$$

"

.

$H$

grable function space. $H^{1}(\Omega)$ is a subspace of $L^{2}(\Omega)$, such that the deriva-

tives of functions in $H^{1}(\Omega)$ are elements of $L^{2}(\Omega)$. Finally the 0 and $D$

subscripts mark the subspace of $H^{1}(\Omega)$ that is constant 0 on the boundary

$\Gamma$, or satisfy the Dirichlet conditions on boundary $\Gamma$ respectively. $\mathbf{H}_{0}^{1}(\Omega)$ and $\mathbf{H}_{D}^{1}(\Omega)$ are their 2 or 3 dimensional vector counterparts. $(\cdot, \cdot)$ is the $L^{2}$ inner product.

For temporally evolving problems, the above mentioned function spaces are extended with a temporal component: $\mathbf{V}_{t} \equiv L^{2}\left(t_{0}, t_{f} ; \mathbf{V}_{D}\right), Q_{t} \equiv \mathcal{D}^{\prime}\left(t_{0}, t_{f} ; Q\right)$, and $E_{t, k} \equiv L^{2}\left(t_{0}, t_{f} ; E_{D, k}\right)$, where $L^{p}\left(t_{0}, t_{f} ; X\right)$ is the space of time dependent functions in a normed space $X$ such that $\int_{t_{0}}^{t_{f}}\|f\|_{X}^{p} \mathrm{~d} t<\infty, 1 \leq p \leq \infty$, and $Q_{t}$ consists of mappings whose $Q$-norm is a distribution in time. The weak form of equations (3), (4), and (5) is defined as: find $\mathbf{u} \in \mathbf{V}_{t}, p \in Q_{t}$, 
and $\xi_{k} \in E_{t, k}$ such that:

$$
\begin{aligned}
\left(\partial_{t} \rho, q\right)+(\nabla \cdot(\rho \mathbf{u}), q) & =0 \\
\left(\partial_{t}(\rho \mathbf{u}), \mathbf{v}\right)+(C(\rho, \mathbf{u}), \mathbf{v})-(p, \nabla \cdot \mathbf{v})+(\boldsymbol{\tau}(\mathbf{u}), \nabla \mathbf{v}) & =(\mathbf{f}, \mathbf{v}), \\
\left(\partial_{t}\left(\rho \xi_{k}\right), \eta_{k}\right)+\left(\nabla \cdot\left(\rho \xi_{k} \mathbf{u}\right), \eta_{k}\right)+\left(\rho D_{k} \nabla \xi_{k}, \nabla \eta_{k}\right) & =\left(S_{k}, \eta_{k}\right),
\end{aligned}
$$

\section{Convection of momentum in variable density flows}

The discretization strategy of $C(\rho, \mathbf{u})$ is a critical feature of flow simulation algorithms, since the convection term should not introduce numerical dissipation [14]. A widely used discretized form for this term is the skewsymmetric formulation, which is preferred for its kinetic energy conserving nature,

$$
C_{\text {skew }}(\rho, \mathbf{u})=(\rho \mathbf{u} \cdot \nabla) \mathbf{u}+\frac{1}{2}(\nabla \cdot(\rho \mathbf{u})) \mathbf{u} .
$$

Below we extend the Energy, Momentum, and Angular momentum Conserving (EMAC) formulation of Charnyi at al. [5] to variable density flows. The following definitions are used: For $\mathbf{u}, \mathbf{v}, \mathbf{w} \in \mathbf{H}^{1}(\Omega)$ we define the trilinear form $b: \mathbf{H}^{1}(\Omega) \times \mathbf{H}^{1}(\Omega) \times \mathbf{H}^{1}(\Omega) \rightarrow \mathbb{R}$ as:

$$
b(\mathbf{u}, \mathbf{v}, \mathbf{w})=(\mathbf{u} \cdot \nabla \mathbf{v}, \mathbf{w}) .
$$

The following identities hold assuming $\mathbf{u} \in \mathbf{H}_{0}^{1}(\Omega)$. They follow directly from integration by parts,

$$
\begin{aligned}
& b(\mathbf{u}, \mathbf{v}, \mathbf{w})=-b(\mathbf{u}, \mathbf{w}, \mathbf{v})-((\nabla \cdot \mathbf{u}) \mathbf{v}, \mathbf{w}), \\
& b(\mathbf{u}, \mathbf{w}, \mathbf{w})=-\frac{1}{2}((\nabla \cdot \mathbf{u}) \mathbf{w}, \mathbf{w}) .
\end{aligned}
$$


Furthermore, by definition, the trilinear form can be also written as:

$$
b(\mathbf{u}, \mathbf{v}, \mathbf{w})=((\nabla \mathbf{v}) \mathbf{u}, \mathbf{w})=\left((\nabla \mathbf{v})^{T} \mathbf{w}, \mathbf{u}\right) .
$$

Additionally the symmetric part of the gradient is denoted as $\nabla_{s} \mathbf{u} \equiv \mathbf{D}(\mathbf{u})=$ $\frac{\nabla \mathbf{u}+(\nabla \mathbf{u})^{T}}{2}$, while the skew-symmetric part is: $\nabla_{n} \mathbf{u} \equiv \frac{\nabla \mathbf{u}-(\nabla \mathbf{u})^{T}}{2}$. For $\mathbf{u}, \mathbf{v} \in$ $\mathbf{H}^{1}(\Omega)$ the following holds,

$$
\begin{aligned}
\left(\nabla_{n} \mathbf{u}\right) \mathbf{v} & =\frac{1}{2}(\nabla \times \mathbf{u}) \times \mathbf{v} \\
(\mathbf{u} \cdot \nabla) \mathbf{u} & =(\nabla \times \mathbf{u}) \times \mathbf{u}+\frac{1}{2} \nabla|\mathbf{u}|^{2} \\
(\nabla \mathbf{u}) \mathbf{u} & =\left(\nabla_{s} \mathbf{u}\right) \mathbf{u}+\left(\nabla_{n} \mathbf{u}\right) \mathbf{u}=\mathbf{D}(\mathbf{u}) \mathbf{u}+\frac{1}{2}(\nabla \times \mathbf{u}) \times \mathbf{u} .
\end{aligned}
$$

Furthermore (22) implies that,

$$
(\mathbf{D}(\mathbf{u}) \mathbf{u}, \mathbf{u})=((\nabla \mathbf{u}) \mathbf{u}, \mathbf{u})=b(\mathbf{u}, \mathbf{u}, \mathbf{u})
$$

Using (20), (21), and (22) we get,

$$
(\mathbf{u} \cdot \nabla) \mathbf{u}=2 \mathbf{D}(\mathbf{u}) \mathbf{u}-\frac{1}{2} \nabla|\mathbf{u}|^{2} .
$$

Next we introduce the notation $\mathbb{A} \equiv \sqrt{\rho} \mathbf{u}$. This change of variables allows us to preserve the symmetric properties used in the incompressible formulation of Charnyi at al. [5]. The nonlinear term can be written as:

$$
C=\nabla \cdot(\mathbb{A} \otimes \mathbb{A})=(\nabla \cdot \mathbb{A}) \mathbb{A}+(\mathbb{A} \cdot \nabla) \mathbb{A}
$$

Using (24) yields the proposed EMAC formulation for variable density flows,

$$
C_{\text {emac }}(\rho, \mathbf{u}) \equiv 2 \mathbf{D}(\mathbb{A}) \mathbb{A}+(\nabla \cdot \mathbb{A}) \mathbb{A}-\frac{1}{2} \nabla|\mathbb{A}|^{2}
$$

These two discretizations of the convective term will be considered for analysis in turbulent conditions for flows with variable density and composition. 


\subsection{Kinetic energy}

The kinetic energy is defined as: $E=\frac{1}{2}(\rho \mathbf{u}, \mathbf{u})=\frac{1}{2} \int_{\Omega} \rho|\mathbf{u}|^{2} \mathrm{~d} \Omega$. We formulate the equation of kinetic energy conservation using the weak form of the momentum equation. Testing (13) with $\mathbf{v}=\mathbf{u}$ yields:

$$
\left(\partial_{t}(\rho \mathbf{u}), \mathbf{u}\right)+(C(\rho, \mathbf{u}), \mathbf{u})-(p, \nabla \cdot \mathbf{u})+(\boldsymbol{\tau}(\mathbf{u}), \nabla \mathbf{u})=(\mathbf{f}, \mathbf{u})
$$

The pressure term of (27) expresses the volumetric work of the hydrodynamic pressure, which vanishes in the incompressible limit and corresponds to the work of dilatation otherwise. (E.g.: the flow accelerating due to heat release.) This can be shown by reformulating the continuity equation (3) as:

$$
\left(\frac{1}{\rho}\left(\partial_{t} \rho+\mathbf{u} \cdot \nabla \rho\right), q\right)=-(q, \nabla \cdot \mathbf{u}) .
$$

Testing (28) with $q=p$ and introducing the notation of material derivative $\frac{D \rho}{D t}=\partial_{t} \rho+\mathbf{u} \cdot \nabla \rho$ gives the term:

$$
-(p, \nabla \cdot \mathbf{u})=\left(\frac{1}{\rho} \frac{D \rho}{D t}, p\right)
$$

Using the common interpretation of the material derivative, this expression indicates, that if the density of fluid particles change, the hydrodynamic pressure is doing work on the fluid. From (27) the temporal evolution of the 
total kinetic energy in the domain $\Omega$ is:

$$
\frac{1}{2} \frac{\mathrm{d}}{\mathrm{d} t}\left\|\rho \mathbf{u}^{2}\right\|=-(C(\rho, \mathbf{u}), \mathbf{u})-\left(\frac{1}{\rho} \frac{D \rho}{D t}, p\right)-\left(2 \mu \mathbf{S}^{\mathbf{D}}(\mathbf{u}), \nabla \mathbf{u}\right)+(\mathbf{f}, \mathbf{u}) .
$$

To study the effect of the discretization strategy on the kinetic energy transport we consider a non-reacting $\left(\frac{D \rho}{D t}=0\right)$, inviscid $(\mu=0)$ flow without body force $(\mathbf{f}=\mathbf{0})$ on a periodic domain. Thus the kinetic energy over the whole periodic domain is preserved by the convective operator if: $(C(\rho, \mathbf{u}), \mathbf{u})=0$.

We can prove that the skew-symmetric formulation of the convective term is kinetic energy preserving, by using (18) to get,

$$
\begin{aligned}
\left(C_{\text {skew }}(\rho, \mathbf{u}), \mathbf{u}\right) & =((\rho \mathbf{u} \cdot \nabla) \mathbf{u}, \mathbf{u})+\left(\frac{1}{2}(\nabla \cdot(\rho \mathbf{u})) \mathbf{u}, \mathbf{u}\right) \\
& =b(\rho \mathbf{u}, \mathbf{u}, \mathbf{u})-b(\rho \mathbf{u}, \mathbf{u}, \mathbf{u})=0
\end{aligned}
$$

For the EMAC formulation the proof is the following:

$$
\left(C_{\text {emac }}(\rho, \mathbf{u}), \mathbf{u}\right)=2\left(\mathbf{D}(\mathbb{A}) \mathbb{A}, \frac{\mathbb{A}}{\sqrt{\rho}}\right)+\left((\nabla \cdot \mathbb{A}) \mathbb{A}, \frac{\mathbb{A}}{\sqrt{\rho}}\right)-\left(\frac{1}{2} \nabla|\mathbb{A}|^{2}, \frac{\mathbb{A}}{\sqrt{\rho}}\right) .
$$

The first term of (32) is transformed using (19) and the definition of the trilinear form,

$$
\begin{aligned}
2\left(\mathbf{D}(\mathbb{A}) \mathbb{A}, \frac{\mathbb{A}}{\sqrt{\rho}}\right) & =\left((\nabla \mathbb{A}) \mathbb{A}, \frac{\mathbb{A}}{\sqrt{\rho}}\right)+\left((\nabla \mathbb{A})^{T} \frac{\mathbb{A}}{\sqrt{\rho}}, \mathbb{A}\right) \\
& =2 b\left(\mathbb{A}, \mathbb{A}, \frac{\mathbb{A}}{\sqrt{\rho}}\right)=2\left((\mathbb{A} \cdot \nabla) \mathbb{A}, \frac{\mathbb{A}}{\sqrt{\rho}}\right) \\
& =2\left((\mathbb{A} \cdot \nabla) \frac{\mathbb{A}}{\sqrt[4]{\rho}} \frac{\mathbb{A}}{\sqrt[4]{\rho}}\right)=2 b\left(\mathbb{A}, \frac{\mathbb{A}}{\sqrt[4]{\rho}}, \frac{\mathbb{A}}{\sqrt[4]{\rho}}\right) .
\end{aligned}
$$

Using (18) the second term of (32) is:

$$
\left((\nabla \cdot \mathbb{A}) \mathbb{A}, \frac{\mathbb{A}}{\sqrt{\rho}}\right)=\left((\nabla \cdot \mathbb{A}) \frac{\mathbb{A}}{\sqrt[4]{\rho}}, \frac{\mathbb{A}}{\sqrt[4]{\rho}}\right)=-2 b\left(\mathbb{A}, \frac{\mathbb{A}}{\sqrt[4]{\rho}}, \frac{\mathbb{A}}{\sqrt[4]{\rho}}\right) .
$$




\subsection{Momentum}

The linear momentum is defined as: $\mathbf{M}=\int_{\Omega} \rho \mathbf{u d} \Omega$. To prove its conservation, we are testing (12) and (13) with $\mathbf{v}=\chi\left(\mathbf{e}_{i}\right)$ and $q=0$, where $\chi\left(\mathbf{e}_{i}\right)$ is a function such that:

$$
\chi\left(\mathbf{e}_{i}\right)= \begin{cases}\mathbf{e}_{i} & \text { on } \hat{\Omega}, \\ \mathbf{g} \in \mathbf{H}_{0}^{1}(\Omega) & \text { on } S=\Omega \backslash \hat{\Omega},\end{cases}
$$

where $\mathbf{g}$ is an arbitrary function satisfying zero boundary conditions, and $\hat{\Omega}$ is a strictly interior subdomain. Testing with this function yields,

$$
\left(\partial_{t}(\rho \mathbf{u}), \mathbf{e}_{i}\right)+\left(C(\rho, \mathbf{u}), \mathbf{e}_{i}\right)-\left(p, \nabla \cdot \mathbf{e}_{i}\right)+\left(\boldsymbol{\tau}(\mathbf{u}), \nabla \mathbf{e}_{i}\right)=\left(\mathbf{f}, \mathbf{e}_{i}\right) .
$$

The terms $\left(p, \nabla \cdot \mathbf{e}_{i}\right)$ and $\left(\boldsymbol{\tau}(\mathbf{u}), \nabla \cdot \mathbf{e}_{i}\right)$ vanish, as $\mathbf{e}_{i}$ is constant. Thus (36) simplifies to:

$$
\frac{\mathrm{d}}{\mathrm{d} t}\left(\rho \mathbf{u}, \mathbf{e}_{i}\right)+\left(C(\rho, \mathbf{u}), \mathbf{e}_{i}\right)=\left(\mathbf{f}, \mathbf{e}_{i}\right)
$$

and linear momentum is conserved if $\left(\mathbf{f}, \mathbf{e}_{i}\right)=0$ and $\left(C(\rho, \mathbf{u}), \mathbf{e}_{i}\right)=0$. Latter term in the skew-symmetric formulation is non-zero. Using (17), the definition of the trilinear form, and the fact that the derivatives of $\mathbf{e}_{i}$ are 
zero, we get:

$$
\begin{aligned}
\left(C_{\text {skew }}(\rho, \mathbf{u}), \mathbf{e}_{i}\right) & =\left((\rho \mathbf{u} \cdot \nabla) \mathbf{u}, \mathbf{e}_{i}\right)+\left(\frac{1}{2}(\nabla \cdot(\rho \mathbf{u})) \mathbf{u}, \mathbf{e}_{i}\right) \\
& =b\left(\rho \mathbf{u}, \mathbf{u}, \mathbf{e}_{i}\right)+\frac{1}{2}\left((\nabla \cdot(\rho \mathbf{u})) \mathbf{u}, \mathbf{e}_{i}\right) \\
& =-b\left(\rho \mathbf{u}, \mathbf{e}_{i}, \mathbf{u}\right)-\frac{1}{2}\left((\nabla \cdot(\rho \mathbf{u})) \mathbf{u}, \mathbf{e}_{i}\right) \\
& =-\frac{1}{2}\left((\nabla \cdot(\rho \mathbf{u})) \mathbf{u}, \mathbf{e}_{i}\right) \neq 0
\end{aligned}
$$

hence the skew-symmetric formulation does not conserve linear momentum. For the EMAC formulation the momentum conservation is proven as follows,

$$
\left(C_{\text {emac }}(\rho, \mathbf{u}), \mathbf{e}_{i}\right)=2\left(\mathbf{D}(\mathbb{A}) \mathbb{A}, \mathbf{e}_{i}\right)+\left((\nabla \cdot \mathbb{A}) \mathbb{A}, \mathbf{e}_{i}\right)-\left(\frac{1}{2} \nabla|\mathbb{A}|^{2}, \mathbf{e}_{i}\right)
$$

The first term of (39) is rearranged using (17) and (19),

$$
\begin{aligned}
2\left(\mathbf{D}(\mathbb{A}) \mathbb{A}, \mathbf{e}_{i}\right) & =\left((\nabla \mathbb{A}) \mathbb{A}, \mathbf{e}_{i}\right)+\left((\nabla \mathbb{A})^{T} \mathbb{A}, \mathbf{e}_{i}\right) \\
& =b\left(\mathbb{A}, \mathbb{A}, \mathbf{e}_{i}\right)+b\left(\mathbf{e}_{i}, \mathbb{A}, \mathbb{A}\right) \\
& =-b\left(\mathbb{A}, \mathbf{e}_{i}, \mathbb{A}\right)-\left((\nabla \cdot \mathbb{A}) \mathbb{A}, \mathbf{e}_{i}\right)-\frac{1}{2}\left(\left(\nabla \cdot \mathbf{e}_{i}\right) \mathbb{A}, \mathbb{A}\right) \\
& =-\left((\nabla \cdot \mathbb{A}) \mathbb{A}, \mathbf{e}_{i}\right),
\end{aligned}
$$

\subsection{Angular momentum}

The angular momentum is defined as: $\mathbf{M}_{\mathbf{x}}=\int_{\Omega} \rho \mathbf{u} \times \mathbf{x d} \Omega$, where $\mathbf{x}$ is the position vector. We are testing (12) and (13) with $\mathbf{v}=\chi\left(\phi_{i}\right)$ and $q=0$, where $\phi_{i} \equiv \mathbf{x} \times \mathbf{e}_{i}$,

$$
\left(\partial_{t}(\rho \mathbf{u}), \phi_{i}\right)+\left(C(\rho, \mathbf{u}), \phi_{i}\right)-\left(p, \nabla \cdot \phi_{i}\right)+\left(\boldsymbol{\tau}(\mathbf{u}), \nabla \phi_{i}\right)=\left(\mathbf{f}, \phi_{i}\right) .
$$


The pressure term vanishes since $\nabla \cdot \phi_{i}=0$, and in inviscid cases without source terms the requirement for angular momentum conservation is $\left(C(\rho, \mathbf{u}), \phi_{i}\right)=0$, similarly to above. For the skew-symmetric formulation, using the same arguments as for (40) yields:

$$
\left(C_{\text {skew }}(\rho, \mathbf{u}), \phi_{i}\right)=-\frac{1}{2}\left((\nabla \cdot(\rho \mathbf{u})) \mathbf{u}, \phi_{i}\right) \neq 0,
$$

thus the skew-symmetric formulation does not conserve angular momentum. For the EMAC formulation,

$$
\left(C_{\text {emac }}(\rho, \mathbf{u}), \phi_{i}\right)=2\left(\mathbf{D}(\mathbb{A}) \mathbb{A}, \phi_{i}\right)+\left((\nabla \cdot \mathbb{A}) \mathbb{A}, \phi_{i}\right)-\left(\frac{1}{2} \nabla|\mathbb{A}|^{2}, \phi_{i}\right),
$$

the last term is 0 by the same argument as for Linear momentum. The remaining terms can be transformed using (19), and (17),

$$
\begin{aligned}
\left(C_{\text {emac }}(\rho, \mathbf{u}), \phi_{i}\right) & =2\left(\mathbf{D}(\mathbb{A}) \mathbb{A}, \phi_{i}\right)+\left((\nabla \cdot \mathbb{A}) \mathbb{A}, \phi_{i}\right) \\
& =b\left(\mathbb{A}, \mathbb{A}, \phi_{i}\right)+b\left(\phi_{i}, \mathbb{A}, \mathbb{A}\right)+\left((\nabla \cdot \mathbb{A}) \mathbb{A}, \phi_{i}\right) \\
& =b\left(\mathbb{A}, \mathbb{A}, \phi_{i}\right)+\left((\nabla \cdot \mathbb{A}) \mathbb{A}, \phi_{i}\right) \\
& =-b\left(\mathbb{A}, \phi_{i}, \mathbb{A}\right)=0,
\end{aligned}
$$

since $\nabla \cdot \phi_{i}=0$. Thus, it is shown, that the EMAC operator conserves also angular momentum.

\section{Numerical methods}

This section introduces a low-dissipation discretization strategy for variable density flows in the low Mach number limit within the finite element context. It includes the spatial discretization of the governing equations, where the stabilization of the pressure in the momentum equation comes from the 
numerical dissipation of the fractional step scheme [14], while stabilized-finite elements are used for scalar transport. An explicit third-order Runge-Kutta temporal scheme is used for the momentum and scalar transport.

\subsection{Spacial discretization}

Finite elements are used to construct the spacial discretization. The following piecewise linear subspaces are approximating the continuous subspaces: $\mathbf{V}_{D, h} \subset \mathbf{V}_{D}, \mathbf{V}_{0, h} \subset \mathbf{V}_{0}, Q_{h} \subset Q, E_{D, k, h} \subset E_{D, k}$, and $E_{0, k, h} \subset E_{0, k}$. The discretized problem is: find $\mathbf{u}_{\mathbf{h}}^{\mathbf{n}+\mathbf{1}} \in \mathbf{V}_{D, h}, p_{h}^{n+1} \in Q_{h}$, and $\xi_{k, h}^{n+1} \in E_{D, k, h}$ such that:

$$
\begin{gathered}
\left(\partial_{t} \rho_{h}, q_{h}\right)+\left(\nabla \cdot\left(\rho_{h} \mathbf{u}_{h}\right), q_{h}\right)=0 \\
\left(\partial_{t}\left(\rho_{h} \mathbf{u}_{h}\right), \mathbf{v}_{h}\right)+\left(C\left(\rho_{h}, \mathbf{u}_{h}\right), \mathbf{v}_{h}\right)-\left(p_{h}, \nabla \cdot \mathbf{v}_{h}\right) \\
+\left(\boldsymbol{\tau}\left(\mathbf{u}_{h}\right), \nabla \mathbf{v}_{h}\right)=\left(\mathbf{f}, \mathbf{v}_{h}\right) \\
\left(\rho_{h} \partial_{t} \xi_{k, h}, \eta_{k, h}\right)+\left(\rho_{h} \mathbf{u}_{h} \cdot \nabla \xi_{k, h}, \eta_{k, h}\right)+\left(\rho D_{k} \nabla \xi_{k, h}, \nabla \eta_{k, h}\right) \\
+\sum_{e=1}^{n_{e l}} \tau_{e}\left(\rho_{h} \mathbf{u}_{h} \cdot \nabla \xi_{k, h}+\nabla \cdot\left(\rho D_{k} \nabla \xi_{k, h}\right)-S_{k}, \mathbf{u}_{h} \cdot \nabla \eta_{k, h}\right)=\left(S_{k}, \eta_{k, h}\right)
\end{gathered}
$$

for all $\left(q_{h}, \mathbf{v}_{h}, \eta_{k, h}\right) \in Q_{h} \times \mathbf{V}_{0, h} \times E_{0, k, h}$. Note, that the scalars are transported using the advective form, and stablilization is introduced where $n_{e l}$ is the number of elements, and $\tau_{e}$ is a local timescale for the stabilization in accordance with Codina et al.[13]. The discretized equations are used in matrix form, hereafter,

$$
\begin{aligned}
\mathrm{M} \partial_{t} \mathcal{D}+\mathrm{D}[\mathcal{D} \mathbf{U}] & =\mathrm{R}_{\mathrm{c}} \\
\mathrm{MI} \partial_{t}[\mathcal{D} \mathbf{U}]+\mathrm{K}_{\mathrm{m}} \mathbf{U}+\mathbf{G P} & =\mathrm{R}_{\mathrm{m}} \\
\mathrm{MD} \partial_{t} \Xi_{k}+\mathrm{K}_{\mathrm{k}} \Xi_{k} & =\mathrm{R}_{\mathrm{k}}
\end{aligned}
$$


We use $a, b$ for the node indices, and $i, j$ for the space indices. The shape function of node $a$ is $N^{a}$. The following notation is adapted in matrix form: $\mathrm{M}^{a b}=\left(N^{b}, N^{a}\right)$ is the mass matrix of the elements, $\mathrm{D}_{j}^{a b}=\left(\partial_{j} N^{b}, N^{a}\right)$ is the discrete divergence operator, $\mathbf{G}_{i}^{a b}=-\left(N^{b}, \partial_{i} N^{a}\right)$ is the discrete gradient operator, and $\mathrm{I}_{i j}=\delta_{i j}$ is the identity tensor, with $\delta_{i j}$ being the Dirac-delta. $\mathcal{D}, \mathbf{U}, \mathrm{P}$, and $\Xi_{k}$ stand for the matrices of density, velocity, pressure, and $k^{\text {th }}$ scalar respectively.

$\mathrm{K}_{\mathrm{m}}=\mathrm{K}_{\mathrm{m}}^{\mathrm{CONV}}+\mathrm{K}_{\mathrm{m}}^{\mathrm{VISC}}$ is the matrix of convective and viscous terms in the momentum equation. The viscous term is discretized as,

$$
\mathrm{K}_{\mathrm{m}}^{\mathrm{VISC}}{ }_{i j}^{a b}=\left(\boldsymbol{\tau}\left(N^{b}\right), \nabla N^{a}\right) \delta_{i j}
$$

while the convective term with the skew-symmetric and EMAC operators are respectively,

$$
\begin{aligned}
\mathrm{K}_{\mathrm{m}, \mathrm{skew}_{i j}}^{\mathrm{CONV}}{ }_{i j} & =\left(\mathcal{D} \mathbf{U} \cdot \nabla N^{b}, N^{a}\right) \delta_{i j}+\frac{1}{2}\left((\nabla \cdot(\mathcal{D} \mathbf{U})) N^{b}, N^{a}\right) \delta_{i j}, \\
\mathrm{~K}_{\mathrm{m}, \mathrm{emac}_{i j}}^{\mathrm{CONV}^{a b}} & =\sqrt{\mathcal{D}}\left[\left(\mathbb{A}_{h} \cdot \nabla N^{b}, N^{a}\right) \delta_{i j}+\left(\partial_{i} N^{b} \mathbb{A}_{h}^{j}, N^{a}\right)\right. \\
& \left.+\left(\left(\nabla \cdot \mathbb{A}_{h}\right) N^{b}, N^{a}\right) \delta_{i j}-\frac{1}{2}\left(\partial_{i} \mathbb{A}_{h}^{j} N^{b}, N^{a}\right)\right] .
\end{aligned}
$$

For the scalar transport, the convective and diffusive terms are represented by $\mathrm{K}_{\mathrm{k}}=\mathrm{K}_{\mathrm{k}}^{\mathrm{CONV}}+\mathrm{K}_{\mathrm{k}}^{\mathrm{DIFF}}$ matrix similarly. The two terms are discretized as,

$$
\begin{aligned}
\mathrm{K}_{\mathrm{k}}^{\text {DIFF }}{ }^{a b} & =\mathcal{D} D_{k}\left(\nabla N^{b}, \nabla N^{a}\right) \\
& -\left(\nabla\left(\mathcal{D} D_{k}\right) \cdot \nabla N^{b}+\mathcal{D} D_{k} \Delta N^{b}, \tau_{e} \mathbf{U} \cdot \nabla N^{a}\right), \\
\mathrm{K}_{\mathrm{k}}^{\text {CONV }}{ }^{a b} & =\mathcal{D}\left(\mathbf{U} \cdot \nabla N^{b}, N^{a}\right)+\mathcal{D}\left(\mathbf{U} \cdot \nabla N^{b}, \tau_{e} \mathbf{U} \cdot \nabla N^{a}\right) .
\end{aligned}
$$


Note the stabilization terms are included in the matrix forms. The residuals of the equations are treated as,

$$
\begin{aligned}
\mathrm{R}_{\mathrm{c}}{ }^{a} & =0, \\
\mathrm{R}_{\mathrm{m} i}^{a} & =\mathbf{f}_{i} N^{a}, \\
\mathrm{R}_{\mathrm{k}}{ }^{a} & =S_{k}\left(N^{a}+\tau_{e} \mathbf{U} \cdot \nabla N^{a}\right) .
\end{aligned}
$$

\subsection{Temporal discretization}

The time integration of the discretised governing equations is done using the standard $3^{\text {rd }}$ order Runge-Kutta scheme. For scalars, the $\ell^{\text {th }}$ Runge-Kutta substep $(\ell=2 . .3)$ is accomplished as:

$$
\Xi_{k}^{\ell}=\Xi_{k}^{n}+\delta t\left(\mathrm{MD}^{n}\right)^{-1} \sum_{m=1}^{\ell} a_{\ell m}\left(-\mathrm{K}_{\mathrm{k}}^{n} \Xi_{k}^{m}+\mathrm{R}_{k}^{n}\right),
$$

where $\delta t$ is the time step. The complete time step uses the linear combination of the right-hand-sides of the substeps,

$$
\Xi_{k}^{n+1}=\Xi_{k}^{n}+\delta t\left(\mathrm{MD}^{n}\right)^{-1} \sum_{\ell=1}^{s} b_{\ell}\left(-\mathrm{K}_{\mathrm{k}}{ }^{n} \Xi_{k}^{\ell}+\mathrm{R}_{k}^{n}\right) .
$$

For the time marching of the velocity field, the fractional step method is incorporated in the Runge-Kutta steps. First the convective, viscous, and force terms are considered to calculate $\mathbf{U}^{\ell, *}$,

$$
\mathbf{U}^{\ell, *}=\mathbf{U}^{n}+\delta t\left(\mathrm{MID}^{n}\right)^{-1} \sum_{m=1}^{\ell} a_{\ell m}\left(-\mathrm{K}_{\mathrm{m}}\left(\mathbf{U}^{m}, \mathcal{D}^{n}\right) \mathbf{U}^{m}+\mathrm{R}_{m}^{n}\right)
$$

Then, the pressure equation is solved utilizing the discrete continuity equation (48) thus ensuring mass conservation,

$$
\mathrm{D}\left[(\mathrm{MI})^{-1} \mathbf{G} \Phi^{\ell}\right]=\frac{1}{c_{\ell} \delta t}\left(\mathrm{D}\left[\mathcal{D}^{n} \mathbf{U}^{\ell, *}\right]+\mathrm{M} \partial_{t} \mathcal{D}^{\ell}-\mathrm{R}_{\mathrm{c}}^{\ell}\right)
$$


where $\Phi=\mathrm{P}+\mathcal{O}(\delta t)$ is the pseudo-pressure: a first order approximation of the pressure. Optionally the pseudo-pressure in the substep can be approximated using the method of Capuano et al.[20]. By omitting the solution of the Poisson equation (Eq.(62)) in the substeps, the computational time is reduced significantly. Finally the velocity field of the next substep is calculated as:

$$
\mathbf{U}^{\ell}=\mathbf{U}^{\ell, *}-c_{\ell} \delta t\left(\mathrm{MID}^{n}\right)^{-1} \mathbf{G} \Phi^{\ell} .
$$

The complete Navier-Stokes time step is calculated similarly using the linear combination of the right-hand-sides to obtain $\mathbf{U}^{n+1, *}$. Note that the computed pseudo-pressure for the time-step ensures mass conservation.

$$
\begin{aligned}
\mathbf{U}^{n+1, *} & =\mathbf{U}^{n}+\delta t\left(\mathrm{MID}^{n}\right)^{-1} \sum_{\ell=1}^{s} b_{\ell}\left(-\mathrm{K}_{\mathrm{m}}\left(\mathbf{U}^{\ell}, \mathcal{D}^{n}\right) \mathbf{U}^{\ell}+\mathrm{R}_{m}^{n}\right), \\
\mathrm{D}\left[(\mathrm{MI})^{-1} \mathbf{G} \Phi^{n+1}\right] & =\frac{1}{\delta t}\left(\mathrm{D}\left[\mathcal{D}^{n} \mathbf{U}^{n+1, *}\right]+\mathrm{MI} \partial_{t} \mathcal{D}^{n+1}-\mathrm{R}_{c}{ }^{n+1}\right) \\
\mathbf{U}^{n+1} & =\mathbf{U}^{n+1, *}-\delta t\left(\mathrm{MID}^{n}\right)^{-1} \mathbf{G} \Phi^{n+1},
\end{aligned}
$$

where $a_{\ell m}, b_{\ell}$, and $c_{\ell}=\sum_{m} a_{\ell m}$ are the Runge-Kutta coefficients. The time derivative of the density is approximated based on the known values,

$$
\partial_{t} \mathcal{D}^{n+1}=\frac{\mathcal{D}^{n}-\mathcal{D}^{n-1}}{\delta t} .
$$

\subsection{Approximation of the discrete Laplacian}

As the use of the discrete Laplacian $\left(\mathrm{D}(\mathrm{MI})^{-1} \mathbf{G}\right)$ is a rather expensive operation, the use of an approximated Laplacian can reduce substantially the computational cost of the algorithm. Hence, the Laplacian is approximated as:

$$
\mathrm{D}(\mathrm{MI})^{-1} \mathbf{G}=\mathrm{L}, \quad \text { with } \quad \mathrm{L}^{a b}=-\left(\nabla N^{b}, \nabla N^{a}\right) .
$$


A stabilizing effect is introduced to the pressure equation by this approximation. Denoting the difference between the discrete and continous operators with $\mathrm{B}=\mathrm{D}(\mathrm{MI})^{-1} \mathbf{G}-\mathrm{L}$, and using the approximation in equations (62) and (65) for the pseudo-pressure,

$$
\begin{aligned}
\mathrm{L} \Phi^{\ell} & =\frac{1}{c_{\ell} \delta t}\left(\mathrm{D}\left[\mathcal{D}^{n} \mathbf{U}^{\ell, *}\right]+\mathrm{M} \partial_{t} \mathcal{D}^{\ell}-\mathrm{R}_{c}^{\ell}\right), \\
\mathrm{L} \Phi^{n+1} & =\frac{1}{\delta t}\left(\mathrm{D}\left[\mathcal{D}^{n} \mathbf{U}^{n+1, *}\right]+\mathrm{MI} \partial_{t} \mathcal{D}^{n+1}-\mathrm{R}_{c}{ }^{n+1}\right) .
\end{aligned}
$$

Furthermore re-arranging (63) into:

$$
\mathcal{D}^{n} \mathbf{U}^{\ell, *}=\mathcal{D}^{n} \mathbf{U}^{\ell}+c_{\ell} \delta t(\mathrm{MI})^{-1} \mathbf{G} \Phi^{\ell},
$$

re-introduced to (69) we obtain,

$$
\begin{aligned}
& c_{\ell} \delta t \mathrm{~L} \Phi^{\ell}=\mathrm{D}\left[\mathcal{D}^{n} \mathbf{U}^{\ell}+c_{\ell} \delta t(\mathrm{MI})^{-1} \mathbf{G} \Phi^{\ell}\right]-\mathrm{R}_{\mathrm{c}}^{\ell}, \\
& \mathrm{M} \partial_{t} \mathcal{D}^{\ell}+\mathrm{D}\left[\mathcal{D}^{n} \mathbf{U}^{\ell}\right]+c_{\ell} \delta t \mathrm{~B}^{\ell}=\mathrm{R}_{c}^{\ell} .
\end{aligned}
$$

Similarly for the time step,

$$
\mathrm{M} \partial_{t} \mathcal{D}^{n+1}+\mathrm{D}\left[\mathcal{D}^{n} \mathbf{U}^{n+1}\right]+\delta t \mathrm{~B} \Phi^{n+1}=\mathrm{R}_{c}{ }^{n+1} .
$$

Codina \& Blasco [21] showed that error introduced by $B \Phi^{n+1}$ is the same order as the pressure interpolation error: $\mathcal{O}\left(h^{k+1}\right)$, where $k$ is the order of the elements. Using the stabilization term in (28), yields a kinetic energy dissipation term of $(\delta t \mathrm{~B} \Phi, \Phi)$. Thus the stabilization term introduces an error of $\mathcal{O}\left(\delta t h^{k+1}\right)$ in the kinetic energy conservation.

\subsection{Overview of the algorithm}

The complete approach is summarised in Algorithm 1. The time step is calculated as the minimum of the time step of the scalar and the momentum 
equations. For the scalar equations, the standard CFL condition is applied, while the self-adaptive strategy of Trias \& Lehmkuhl [22] is used for the momentum equations. Then the scalars are transported using stablilized finite elements, and the new velocity field is calculated with the fractional step method. For the momentum transport the convective operator is assembled according to (52) or (53) for the skew-symmetric and EMAC discretizations respectively using the density $\left(\mathcal{D}^{n}\right)$ of the previous time step, and velocity $\left(\mathrm{U}^{n}\right)$ of the substeps. There is no need to solve a transport equation for $\mathbb{A}$. 


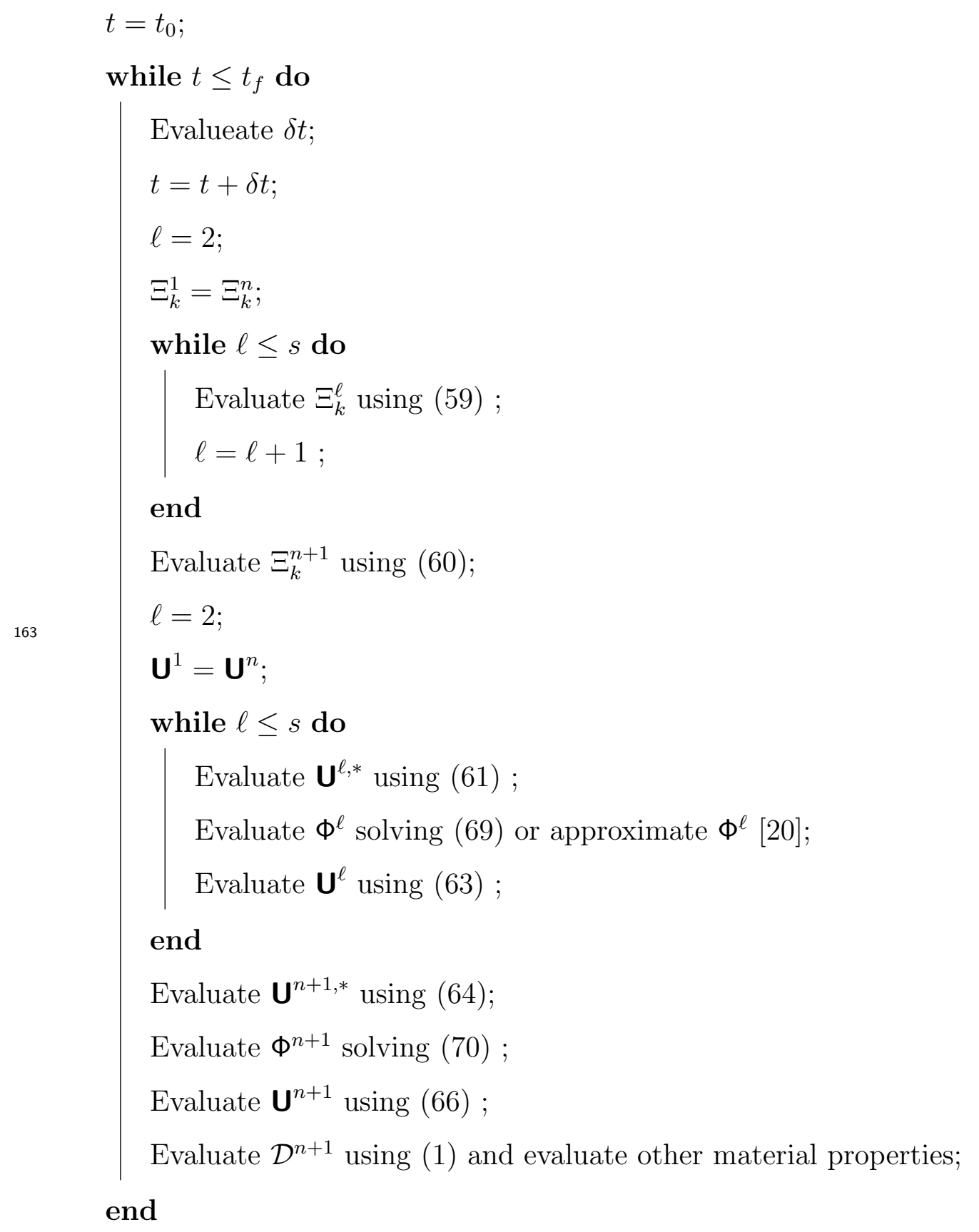

Algorithm 1: Overview of the algorithm 


\section{Numerical tests}

The developed algorithm has been extensively tested in incompressible conditions. A set of dedicated test cases has been published in the recent work of Lehmkuhl et al. [14]. The included tests are: the inviscid and viscous Taylor-Green vortex, a turbulent channel flow, flow past a sphere, and flow around an Ahmed body. Using the inviscid Taylor-Green vortex, it has been shown, that the kinetic energy dissipation introduced by the numerical scheme is indeed scaling as $\mathcal{O}\left(\delta t h^{k+1}\right)$. It is important to note, that the same code is used in the present study by adding the scalar transport equations, and the state dependent material properties, and by including the $\partial_{t} \mathcal{D}$ term in (69) and (70). Thus the results of Lehmkuhl et al. [14] are retained in the incompressible limit with the present formulation. This section presents three application cases with increasing complexity to evaluate the proposed low Mach number discretization strategy for thermally coupled flows subjected to chemical reactions. The first test case corresponds to an anisothermal turbulent channel flow at constant composition with a relatively low density ratio. The second case is a turbulent premixed flame, that is represented by the transport of a reactive scalar also referred as progress variable that describes the thermochemical state of the flame. Finally, the third case corresponds to a turbulent diffusion flame, where a passive scalar also referred as mixture fraction is added as an additional variable to the progress variable to account for the effects of fuel/air mixing. Further details of these modelling approaches and description of the subgrid terms required for LES are given in the corresponding subsections. 


\subsection{Anisothermal channel flow}

The effect of density gradients is tested in an isolated manner with the anisothermal channel flow case of Toutant and Bataille [23] using LES with Favre-averaged quantities [1]. Fig.1 illustrates the domain, which has the dimensions $L_{x}=2 \pi h, L_{y}=2 h$, and $L_{z}=\pi h$ in the streamwise, wallnormal, and spanwise directions respectively, where $h=1 \mathrm{~m}$ was chosen as the channel half-height. Toutant and Bataille [23] showed, that the two point correlations diminish over these distances, and these dimensions can be considered adequate for the problem. The domain is periodic in the spanwise and streamwise directions.

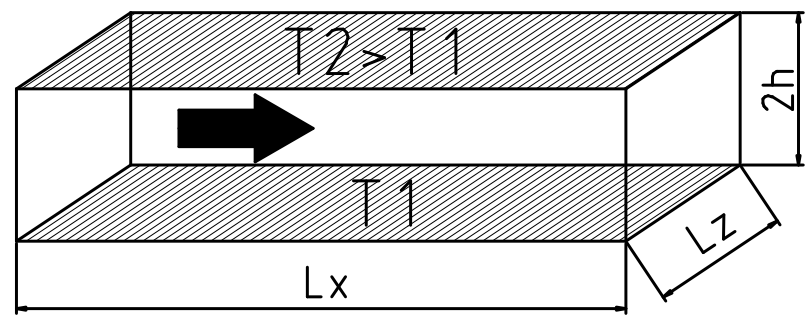

Figure 1: Channel geometry

In this case, the only transported scalar is the temperature. The governing equation substituting (5) using Favre-averaged quantities is:

$$
c_{p} \bar{\rho} \partial_{t} \widetilde{T}+c_{p} \bar{\rho} \widetilde{\mathbf{u}} \cdot \nabla \widetilde{T}+\nabla \cdot\left(-\left(\lambda+\frac{c_{p} \mu^{S G S}}{P r_{t}}\right) \nabla \widetilde{T}\right)=\frac{\mathrm{d} P_{0}}{\mathrm{~d} t},
$$

where $\lambda$ is the thermal conductivity, and $c_{p}=1005 \frac{\mathrm{J}}{\mathrm{kgK}}$ is the specific heat. The material properties are representing realistic gas-like behaviour. The filtering of the continuity and momentum equations (3) and (4) is taken using a standard LES closure based on Boussinesq [1] with the subgrid scale turbulent viscosity given by Vreman [24]. Here, the model constant is set to 
$C=0.1$, as it is the value recommended by the author for complex cases. The equation of state (1) is used with a specific gas constant of $R=\frac{R_{u}}{W}=287 \frac{\mathrm{J}}{\mathrm{kgK}}$, the dynamic viscosity is set by Sutherland's law: $\mu=\frac{T^{3 / 2}}{T+111 \mathrm{~K}} 1.461 \cdot 10^{-6} \frac{\mathrm{Pas}}{\sqrt{\mathrm{K}}}$, the thermal conductivity is calculated using a constant molecular Prandtl number: $\lambda=\frac{\mu c_{p}}{P r}$ with $\operatorname{Pr}=0.71$, and a turbulent Prandtl number of $P r_{t}=$ 0.9 is set as proposed by Toutant and Bataille [23].

The mean flow is induced by applying a constant artificial pressure gradient $\left(\mathbf{f}_{x}\right)$ in the $x$ direction. Following the convention of incompressible channel simulations the flow is characterised by the wall Reynolds number, however the material properties are evaluated at the corresponding wall temperature,

$$
R e_{\tau}=\frac{\rho_{w} u_{\tau} h}{\mu_{w}}
$$

where " $w "$ indicates the wall state $\left(\rho_{w}=\rho\left(P_{0}, T_{w}\right), \mu_{w}=\mu\left(T_{w}\right)\right)$, and $u_{\tau}$ is the friction velocity: $u_{\tau}=\sqrt{\frac{\left\langle\tau_{w}\right\rangle}{\rho_{w}}}$ with $\left\langle\tau_{w}\right\rangle$ being the mean wall shear stress calculated as:

$$
\left\langle\tau_{w}\right\rangle=\frac{1}{\Delta t} \sum_{t_{0}}^{t_{0}+\Delta t} \frac{\left|\mathbf{F}_{w, x}^{n}\right|}{A_{w}} \delta t^{n},
$$

where $\mathbf{F}_{w, x}^{n}$ is the friction force exerted on the wall in the streamwise direction at time step $n, A_{w}$ is the wall surface, $\Delta t$ is length of the averaging interval, and $\delta t^{n}$ is the time step size.

The two walls have different wall Reynolds numbers for non-unity temperature ratios $(T r)$. The usual approach to reach different temperature ratios is keeping the cold wall at room temperature while increasing the temperature of the hot wall. If Sutherland's law is applied, the viscosity at the hot wall increases, and the wall Reynolds number decreases. However, the cases can 
be characterised by the average wall Reynolds number,

$$
R e_{\tau}=\frac{R e_{\tau, c}+R e_{\tau, h}}{2}
$$

"c" and " $h "$ indicating the cold and hot walls respectively. There are numerous approaches in the literature for comparing different temperature ratios. The DNS database considered in this work imposes a volumetric force term such that the average wall Reynolds number is defined $\left(R e_{\tau}=395\right)[23]$. The force term is expressed as:

$$
\mathbf{f}_{x}=\frac{\tau_{c}+\tau_{h}}{2 h}=\frac{\frac{R e_{\tau, c}^{2} \mu_{c}^{2}}{\rho_{c}}+\frac{R e_{\tau, h}^{2} \mu_{h}^{2}}{\rho_{h}}}{2 h^{3}} .
$$

To calculate the force term in the $T r=2$ case, the two wall Reynolds numbers are taken a priori from the DNS [23] as: $R e_{\tau, c}=R e_{\tau, c}^{D N S}=235$ and $R e_{\tau, h}=$ $R e_{\tau, h}^{D N S}=565$. A structured hexahedral mesh is applied with homogeneous size in $x$ and $z$. The mesh is refined in the wall-normal direction according to:

$$
y_{i}=h\left\{1+\frac{1}{\alpha} \tanh \left[\operatorname{atanh}(\alpha)\left(\frac{2 i}{N_{e y}}-1\right)\right]\right\}, \quad i=0 . . N_{e y}
$$

where $N_{e y}$ is the number of elements in $y$, and $\alpha$ is a parameter expressing the level of refinement. A coarse and a fine mesh are considered to evaluate the effects of resolution on the discretization strategy and the parameters of relevance for each mesh are listed in Tab.1.

Finally, the temperature scaling is based on the wall heat flux $\left(\dot{q}_{w}\right)$ and the friction temperature can be defined as:

$$
T_{\tau}=\frac{\dot{q}_{w}}{\rho_{w} c_{p} u_{\tau}}
$$




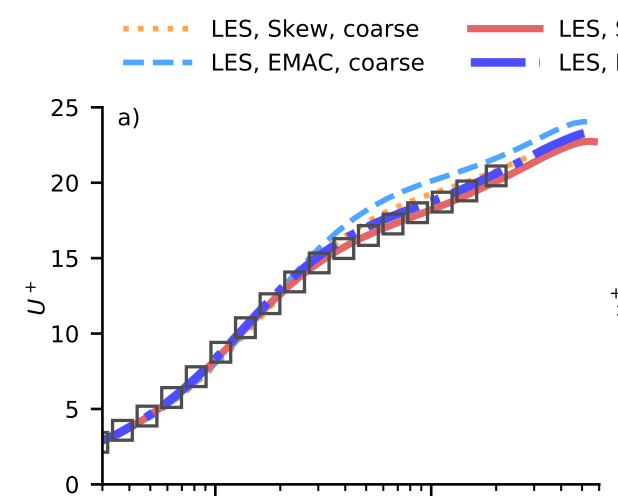

LES, Skew, fine

$\square \quad$ Toutant et al. (2013)
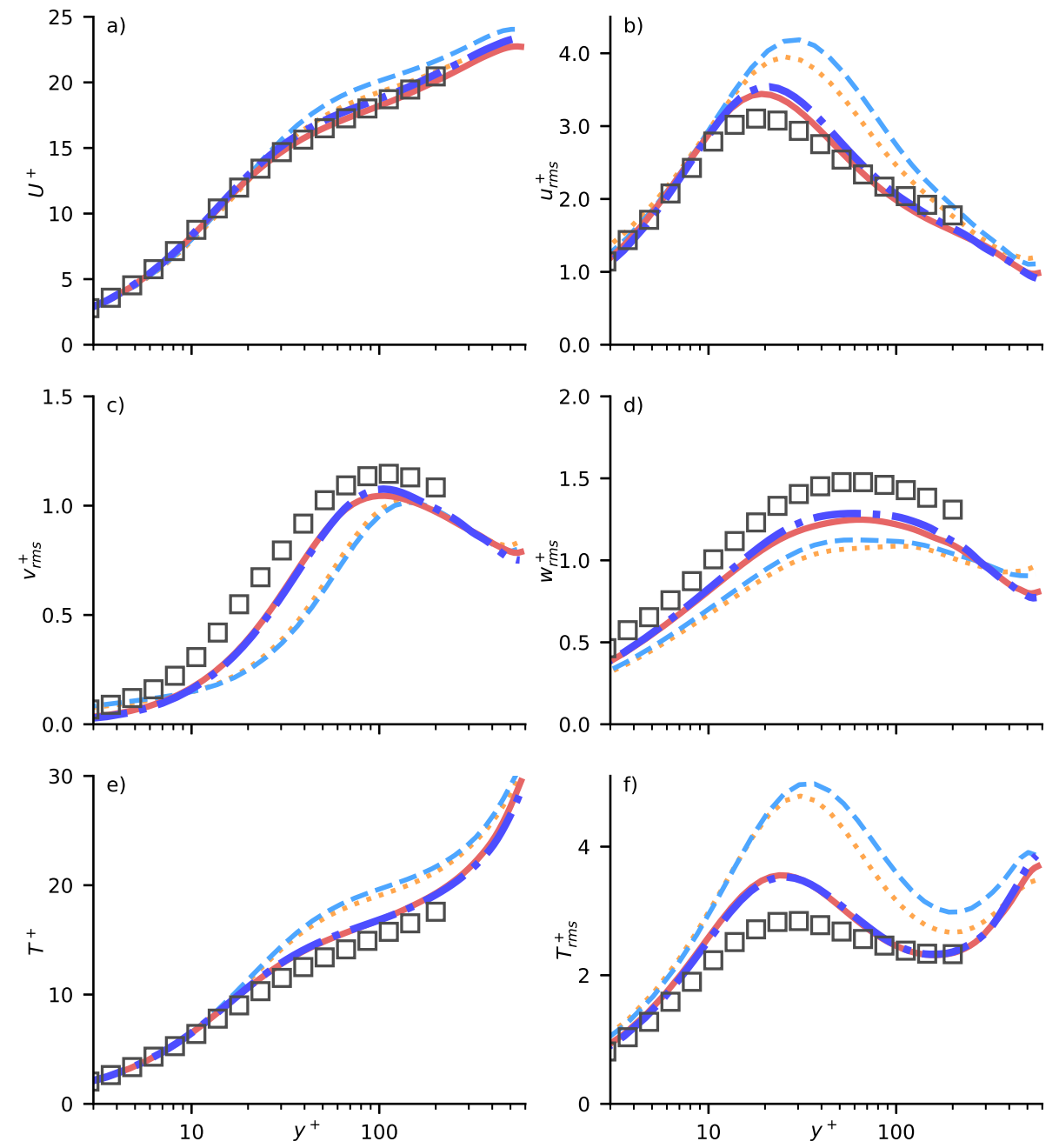

Figure 2: Comparison of mean and RMS properties of the case: $\operatorname{Re}_{\tau}=395, \operatorname{Tr}=2$ on the cold side, a: mean stream-wise velocity, b: stream-wise velocity RMS, c: wallnormal velocity RMS, d: span-wise velocity RMS, e: mean temperature $\left(T^{+}=\frac{T_{w}-\langle T\rangle}{T_{\tau}}\right)$, f: temperature RMS; squares: DNS of Toutant and Bataille [23] (truncated at $y+=200$ ) , dashed blue line: EMAC on coarse mesh, dotted orange line: skew-symmetric on coarse mesh, dash-dotted blue line: EMAC on fine mesh, solid red line: skew-symmetric on fine mesh 

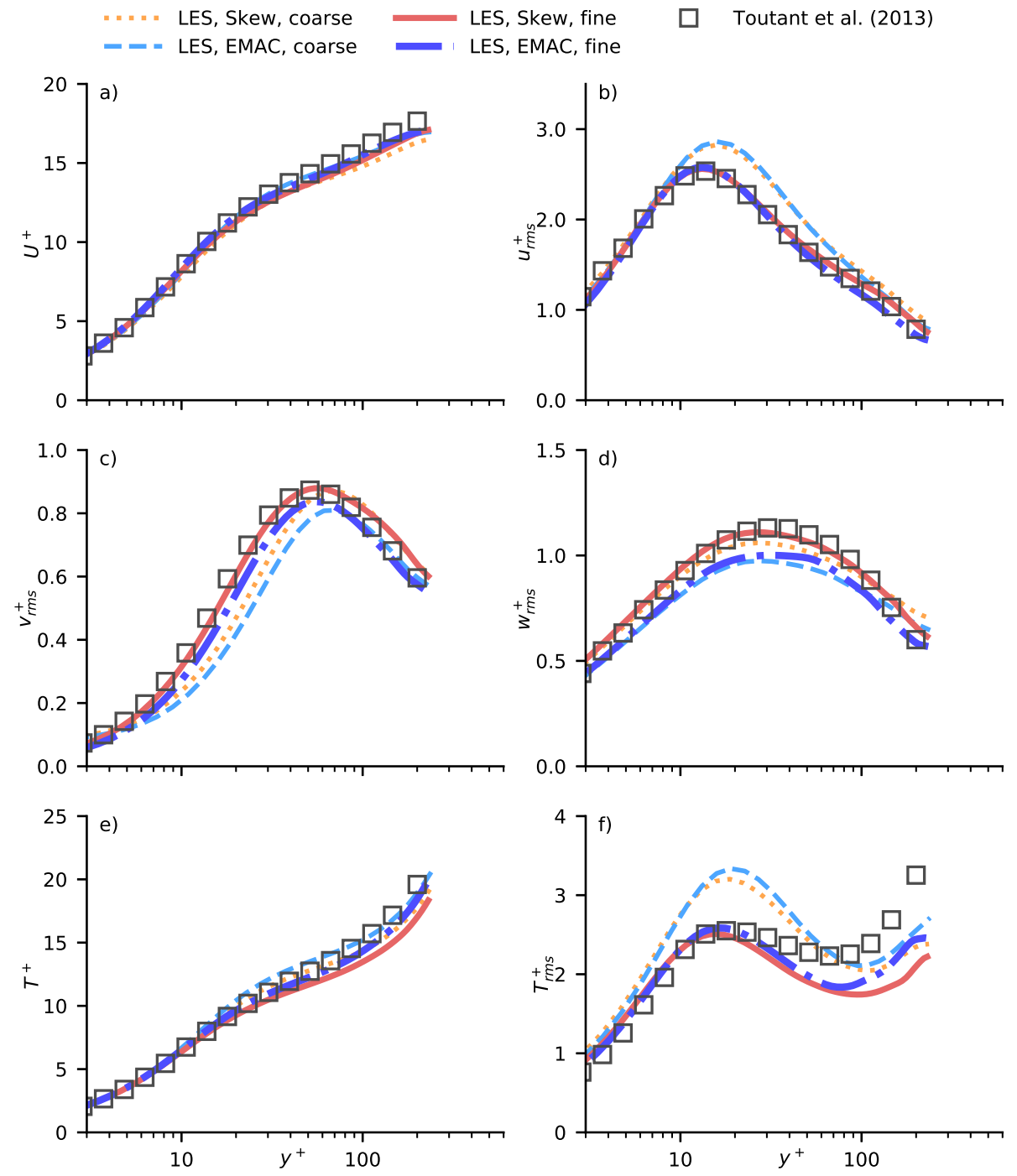

Figure 3: Comparison of mean and RMS properties of the case: $\operatorname{Re} e_{\tau}=395, \operatorname{Tr}=2$ on the hot side, a: mean stream-wise velocity, b: stream-wise velocity RMS, c: wall-normal velocity RMS, d: span-wise velocity RMS, e: mean temperature $\left(T^{+}=\frac{T_{w}-\langle T\rangle}{T_{\tau}}\right)$, f: temperature RMS; squares: DNS of Toutant and Bataille [23] (truncated at $y+=200$ ), dashed blue line: EMAC on coarse mesh, dotted orange line: skew-symmetric on coarse mesh, dashdotted blue line: EMAC on fine mesh, solid red line: skew-symmetric on fine mesh 


\begin{tabular}{l|ccccccccr} 
& $N_{e x}$ & $N_{e y}$ & $N_{e z}$ & $\alpha$ & $\Delta_{x}^{+}$ & $\Delta_{y, \min }^{+}$ & $\Delta_{y, \max }^{+}$ & $\Delta_{z}^{+}$ & DoF \\
\hline Coarse & 70 & 66 & 39 & 0.9933 & 35.5 & 0.50 & 34.2 & 31.8 & $180 \mathrm{k}$ \\
Fine & 165 & 123 & 82 & 0.9830 & 15.0 & 0.54 & 15.5 & 15.1 & $1.66 \mathrm{M}$
\end{tabular}

Table 1: Channel flow mesh size based on $R e_{\tau}=395$

Results for the mean and RMS profiles of the present LES simulations with the DNS of Toutant and Bataille [23] are shown in Figs. 2 and 3 for comparison. The plots indicate a good level of correlation overall with the reference data for the cold and hot sides of the channel. In particular, the results in the cold side are in good agreement for the first-order statistics (normalized streamwise velocity and temperature), while the second-order statistics show a higher dependency with the mesh resolution. On the hot side, the correlation with the reference data is substantially improved when compared to the cold side, and show less dependency on the resolution. The reason behind this different behaviour can be explained by the flow Reynolds number. The flow in the cold side is characterised by higher density than the hot side, and hence, it features a higher Reynolds number. As the mesh was constructed with the same refinement law along the wall-normal direction, the momentum and thermal boundary layer thicknesses are not as well resolved on the cold side as on the hot one. The results also indicate, that there are no significant differences between the skew-symmetric and the EMAC discretizations under these conditions. The wall Reynolds numbers predicted by the present work are listed in Tab.2, and they agree well with the DNS values in all cases. 


\begin{tabular}{c|ccccc} 
& EMAC, coarse & Skew, coarse & EMAC, fine & Skew, fine & DNS[23] \\
\hline Cold & $553(-2.1 \%)$ & $560(-0.9 \%)$ & $571(+1.1 \%)$ & $580(+2.7 \%)$ & 565 \\
Hot & $239(+1.7 \%)$ & $236(+0.4 \%)$ & $238(+1.3 \%)$ & $235(0 \%)$ & 235
\end{tabular}

Table 2: Wall Reynolds numbers on the hot and cold sides at different cases, and the relative error compared to DNS[23]

\subsection{Turbulent premixed swirling flame}

The second test case corresponds to a technically premixed turbulent flame in a swirl-stabilized bluff-body configuration also known as PRECCINSTA burner. The operating point to be investigated corresponds to an equivalence ratio of $\phi=0.75$ that burns in stable operation at Reynolds number $R e=35000$ (based on the swirler outer diameter). Experimental measurements of this burner [25] and previous LES results [26] suggest the perfectly premixed assumption holds for this operating point and the burning of a homogeneous methane/air mixture can be considered to describe the reacting process. The flame exhibits a constant temperature ratio of $\operatorname{Tr} \approx 6$ with fluctuations around the adiabatic unstretched value caused by turbulence/chemistry interactions. This test poses an additional degree of complexity as the density ratio is substantially increased respect to the previous case, and small fluctuations of density will appear influencing the turbulent burning velocity, which is the key element to be predicted here. Furthermore the heat release in the reacting layer causes thermal dilatation, that effectively acts as a volumetric momentum source through the $\partial_{t} \mathcal{D}$ term. The results available for this operating point correspond to mean and fluctuations of velocity, and those will be used for comparison with the proposed 
discretization strategies.

The turbulent combustion model used for this problem is based on flamelet modelling. The governing equations of the problem in the context of LES using Favre-average filtering include the scalar equations for enthalpy $(\widetilde{h})$, scaled progress variable $(\widetilde{C})$, and its subscale variance $\left(C_{v}\right)$, where the notation $(\stackrel{\sim}{-})$ is used for density-weighted variables. The resulting system of equations for scalar transport reads:

$$
\begin{aligned}
\partial_{t}(\bar{\rho} \widetilde{h})+\nabla \cdot(\bar{\rho} \widetilde{h} \widetilde{\mathbf{u}})+\nabla \cdot \mathbf{q} & =\frac{\mathrm{d} P_{0}}{\mathrm{~d} t}, \\
\partial_{t}(\bar{\rho} \widetilde{C})+\nabla \cdot(\bar{\rho} \widetilde{C} \widetilde{\mathbf{u}})+\nabla \cdot \Phi_{C} & =\overline{\dot{\omega}_{C}}, \\
\partial_{t}\left(\bar{\rho} C_{v}\right)+\nabla \cdot\left(\bar{\rho} C_{v} \widetilde{\mathbf{u}}\right)+\nabla \cdot \boldsymbol{\Phi}_{C_{v}} & =-\bar{\rho} \chi_{C_{v}}^{S G S}-2 \boldsymbol{\Phi}_{C}^{S G S} \cdot \nabla \widetilde{C}+\overline{\dot{\omega}}_{C_{v}},
\end{aligned}
$$

where the filtered continuity and momentum equations, as well as the subgrid scale turbulent viscosity corresponds to the ones of the previous case. Here, the constant of the Vreman model is also set to $\mathrm{C}=0.1$, as before. The diffusive fluxes are: : $\mathbf{q}=-\bar{\rho}\left(D+D^{S G S}\right) \nabla \widetilde{h}, \boldsymbol{\Phi}_{C}=-\bar{\rho}\left(D+D^{S G S}\right) \nabla \widetilde{C}$, $\boldsymbol{\Phi}_{C_{v}}=-\bar{\rho}\left(D+D^{S G S}\right) \nabla C_{v}$. The subscale Schmidt number is: $S c^{S G S}=0.7$.

The flamelet database was generated with the GRI3.0 mechanism [27] using a single unstretched adiabatic premixed flamelet at the global equivalence ratio of $\phi=0.75$. In this case the $\beta$-FPDF was applied on the scaled progress variable to account for turbulence-chemistry interaction [28].

The computation domain and measurement locations are presented in Fig.4. The domain includes the plenum, swirler and combustion chamber. A hybrid mesh of $4 \mathrm{M}$ DoF is applied with boundary layer refinement on the walls and tetrahedral mesh in the bulk flow. The base mesh size is $1.5 \mathrm{~mm}$ with $1 \mathrm{~mm}$ refinement in the combustion chamber up to $55 \mathrm{~mm}$ from the 


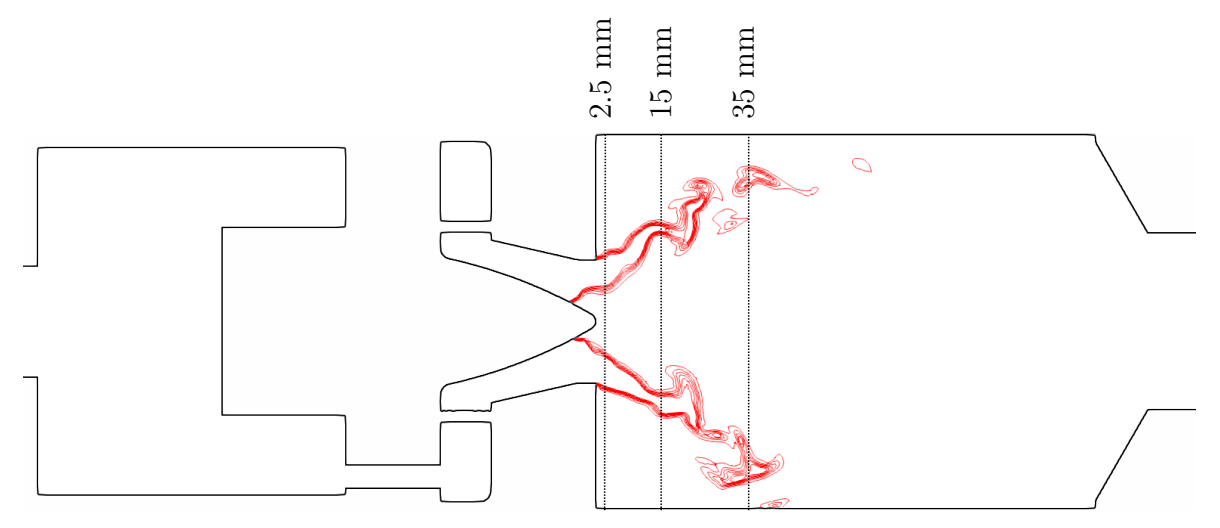

Figure 4: Sketch of domain configuration with measurement locations on top flame visualization with temperature iso-lines: $T \in\{800,1000,1200,1600,1800,2000\} \mathrm{K}$ using EMAC discretization

swirler outlet. Another refinement region with mean mesh size of $0.5 \mathrm{~mm}$ is enclosing the reacting layers. For this problem a single and optimised mesh is used to evaluate the low Mach discretization strategy and the two discretization strategies for the convective term.

The numerical results are compared to the measurements of Meier et al.[25] and are shown in Fig.5 and Fig.6 for analysis. The results indicate the proposed discretization strategy with the two convective term operators provide reasonably good correlation with the experimental data. At upstream locations, the mean values of the three components of the velocity match fairly well the measurements, see first column of Fig.5. The flow in this location, is highly influenced by the anchoring of the flame between the central and corner recirculation zones. The flame is located within the refinement region, and the prediction of velocity is in good agreement. As the flow develops downstream and the mesh is coarsened, the differences between the results 
with the two operators increase. The case with EMAC discretization predicts better the magnitude of the reverse flow of the central recirculation zone, and also the peak values of the axial velocity at the two downstream locations, see Fig. 5 a,b. However, the trends are well predicted by the two operators and show the validity of the proposed low dissipation strategy to solve complex reacting flows. The fluctuating components show the impact of the discretization of the convective term more clearly. While the predicted RMS values at the upstream location are rather similar, the impact of the EMAC discretization at downstream locations is evident, see Fig.6. The EMAC approximation, with enhanced conservative properties with respect to the skew-symmetric operator, show better prediction of the Reynolds stresses in regions with lower resolution.

The relation between the presence of heat release and the worse performance of the skew-symmetric operator can be better understood considering (38). The error in momentum conservation is proportional to $-\nabla \cdot(\rho \mathbf{u})$, which is equal to $\partial_{t} \rho$ in accordance with mass conservation. Thus in the presence of unsteady reacting layers $\left(\left|\partial_{t} \rho\right| \gg 0\right)$, the error introduced by the skew-symmetric discretization is magnified. The EMAC discretization does not have this error. This difference between the two operators is apparent in the downstream measurement locations.

\subsection{DLR-A non-premixed jet flame}

The final case for validation corresponds to a turbulent jet diffusion flame in a low-velocity coflow. This case incorporates certain aspects of the previous case, as it requires solving a reacting flow field subjected to density variations caused by heat release. However in this case, these variations 

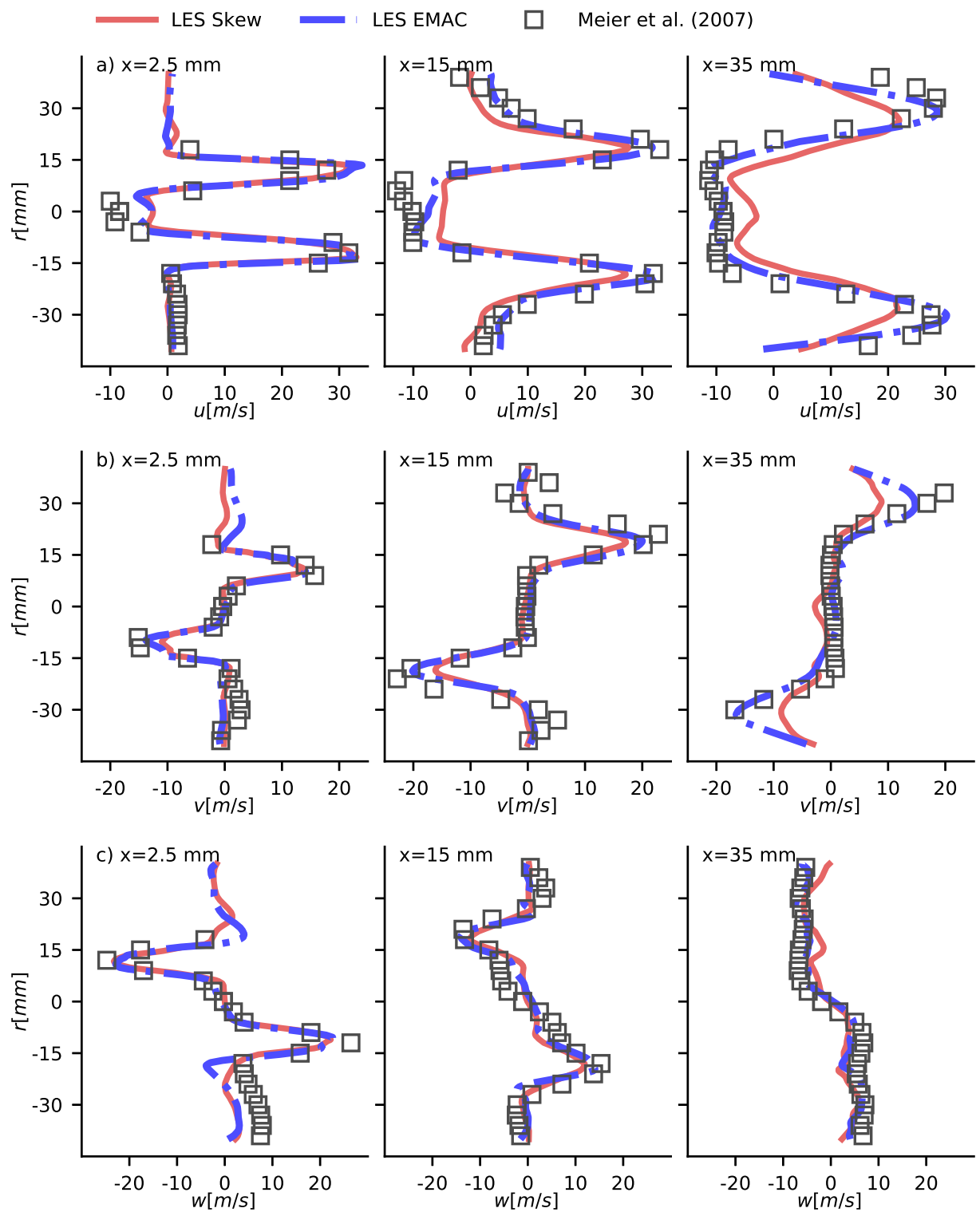

Figure 5: Comparison of mean velocity profiles in the PRECCINSTA burner at three axial locations: $x \in\{2.5,15,35\} \mathrm{mm}$; a: mean axial velocity, b: mean radial velocity, c: mean tangential velocity; squares: measurement of Meier et al. [25], dash-dotted blue line: EMAC, solid red line: skew-symmetric 

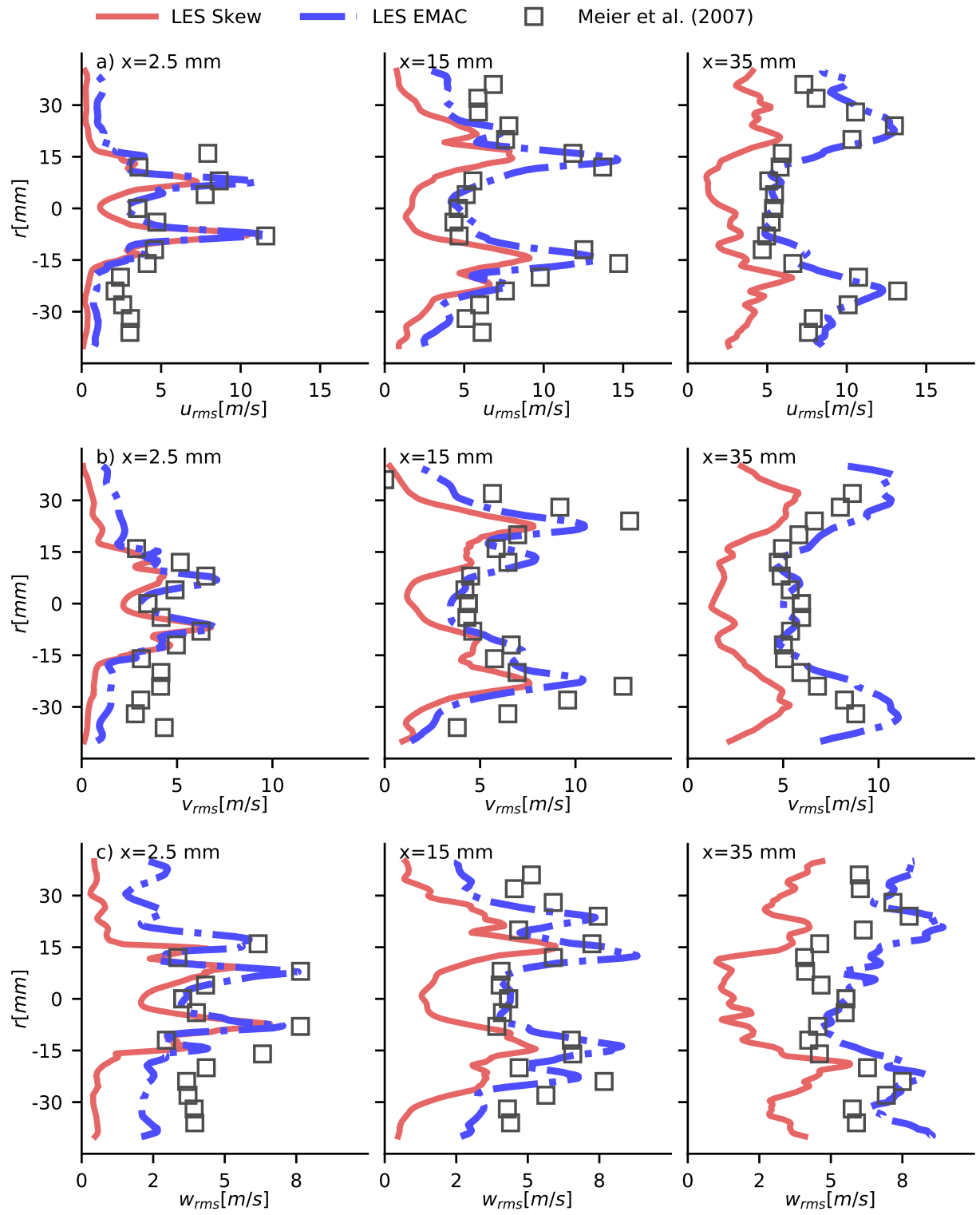

Figure 6: Comparison of RMS velocity profiles in the PRECCINSTA burner at three axial locations: $x \in\{2.5,15,35\} \mathrm{mm}$; a: axial velocity RMS, b: radial velocity RMS, c: tangential velocity RMS; squares: measurement of Meier et al. [25], dash-dotted blue line: EMAC, solid red line: skew-symmetric 
are caused by differences in fuel/air concentration and mixing, so different burning and combustion characteristics are expected in this problem. While in the previous case, the density variation is function of the progress variable ranging from unreacted to fully reacted mixture, the density is direct function of the fuel/air ratio and the progress variable in this problem. In diffusion-dominated conditions, composition variations can be described by the use of an additional transport equation, that is the mixture fraction. Note that fluctuations of mixture fraction are expected to play an important role here as the thermochemical state of the flow is characterised by two controlling variables. This test represents the second fundamental type of regime found in combustion applications, and it has great relevance in practical applications.

The test case considered here is the well-established DLR-A jet flame for which reliable experimental data was generated by DLR and Sandia Labs. [29]. This is a turbulent round jet of a fuel mixture of $\mathrm{CH}_{4} / \mathrm{H}_{2} / \mathrm{N}_{2}$ burning in a low-velocity air coflow. The fuel nozzle has a diameter of $8 \mathrm{~mm}$, the jet is composed of $22.1 \% \mathrm{CH}_{4}, 33.2 \% \mathrm{H}_{2}$, and $44.7 \% \mathrm{~N}_{2}$ by volume, and is supplied at a Reynolds number of 15200 . The temperature ratio at stoichiometric conditions is $T r_{s t}=7$, which is quite similar to the previous case, though this temperature changes also with the mixture fraction to account for fuel/air mixing. The results available for this operating point correspond to mean and fluctuations of velocity, mixture fraction and temperature, and those will be used for comparison with the proposed discretization strategies.

A flamelet model based on diffusion flamelets is used to describe the combustion process in this case, and the filtered scalar transport equations 
to be solved along with Eqs. (3) and (4) reads:

$$
\begin{aligned}
\partial_{t}(\bar{\rho} \tilde{h})+\nabla \cdot(\bar{\rho} \widetilde{h} \widetilde{\mathbf{u}})+\nabla \cdot \mathbf{q} & =\frac{\mathrm{d} P_{0}}{\mathrm{~d} t}, \\
\partial_{t}(\bar{\rho} \widetilde{Z})+\nabla \cdot(\bar{\rho} \widetilde{Z} \widetilde{\mathbf{u}})+\nabla \cdot \Phi_{Z} & =0, \\
\partial_{t}\left(\bar{\rho} Z_{v}\right)+\nabla \cdot\left(\bar{\rho} Z_{v} \widetilde{\mathbf{u}}\right)+\nabla \cdot \Phi_{Z_{v}} & =-\bar{\rho} \chi_{Z_{v}}^{S G S}-2 \boldsymbol{\Phi}_{Z}^{S G S} \cdot \nabla \widetilde{Z}, \\
\partial_{t}\left(\bar{\rho} \widetilde{Y}_{c}\right)+\nabla \cdot\left(\bar{\rho} \widetilde{Y_{c}} \widetilde{\mathbf{u}}\right)+\nabla \cdot \boldsymbol{\Phi}_{Y_{c}} & =\overline{\dot{\omega}_{Y_{c}}},
\end{aligned}
$$

where $h, Z, Z_{v}$, and $Y_{c}$ are the enthalpy, filtered mixture fraction, subscale mixture fraction variance, and filtered progress variable respectively, with diffusive fluxes: $\mathbf{q}=-\bar{\rho}\left(D+D^{S G S}\right) \nabla \widetilde{h}, \mathbf{\Phi}_{Z}=-\bar{\rho}\left(D+D^{S G S}\right) \nabla \widetilde{Z}, \mathbf{\Phi}_{Z_{v}}=$ $-\bar{\rho}\left(D+D^{S G S}\right) \nabla Z_{v}, \boldsymbol{\Phi}_{Y_{c}}=-\bar{\rho}\left(D+D^{S G S}\right) \nabla \widetilde{Y}_{c}$. The molecular diffusivity is $\bar{\rho} D=\frac{\lambda}{c_{p}}$ assuming unity Lewis number, and $\bar{\rho} D^{S G S}=\frac{\mu^{S G S}}{S c_{t}}$ is the subscale diffusivity, where the subscale Schmidt number is constant: $S c^{S G S}=0.7$. The subscale mixture fraction variance has a dissipation term: $\chi_{Z_{v}}^{S G S}=2 \frac{Z_{v}}{\tau_{t}}$ associated with the time scale of the subgrid turbulence: $\tau_{t}=\left(C_{\epsilon}^{2} \nu_{t} \frac{|\mathbf{S}|^{2}}{\Delta^{2}}\right)^{-1 / 3}$ where $C_{\epsilon}=3.24$ is a modelling constant of the subgrid model, $\nu_{t}$ is the subgrid viscosity, $S$ is the strain rate tensor, and $\Delta$ is the element size.

Opposing diffusion flamelets are computed using the GRI3.0 mechanism [27] for generating the flamelet tables. The turbulence-chemistry interaction is considered with $\beta$-function as the Filtered Probability Density Function (FPDF) of the mixture fraction. The necessary material properties, and the source term of the progress variable are integrated with the FPDF a-priori and tabulated as function of $\widetilde{Z}, Z_{v}$, and $\widetilde{C}=\frac{\widetilde{Y_{c}}-\widetilde{Y_{c}^{0}}\left(\widetilde{Z}, Z_{v}\right)}{\widetilde{Y_{c}^{e q}}\left(\widetilde{Z}, Z_{v}\right)-\widetilde{Y_{c}^{0}}\left(\widetilde{Z}, Z_{v}\right)}$, where $\widetilde{C}$ is the scaled progress variable, $\widetilde{Y_{c}^{0}}$ and $\widetilde{Y_{c}^{e q}}$ are the minimum and maximum of progress variable at given $\widetilde{Z}$ and $Z_{v}$.

Two unstructured meshes are used to simulate this case, named coarse 


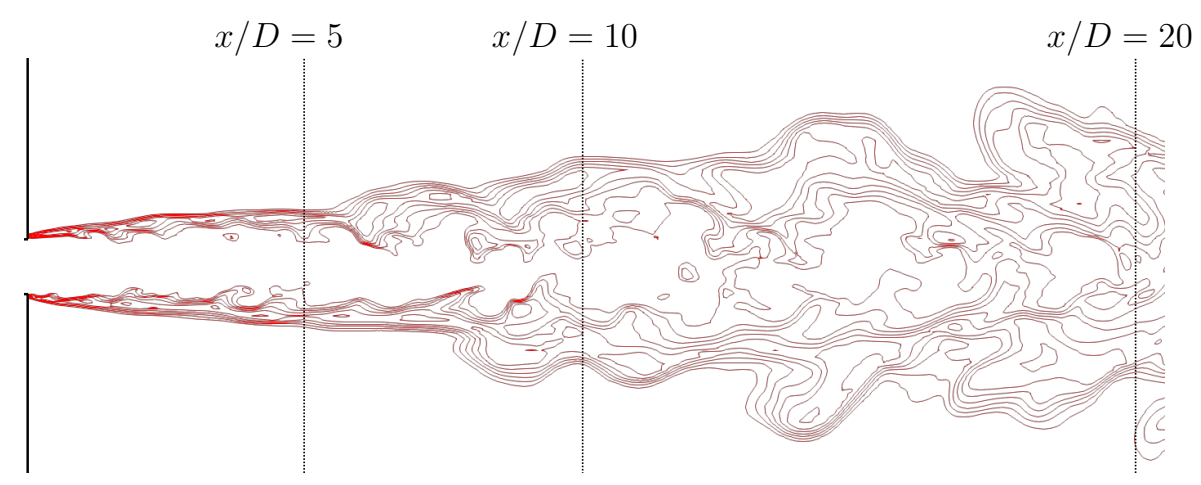

Figure 7: DLR-A jet flame measurement locations, the jet is visualized with temperature iso-lines: $T \in\{800,1000,1200,1600,1800,2000\} \mathrm{K}$ on the fine mesh using EMAC discretization

and fine. The jet axis is in the $x$ direction. A zoomed section of the computational domain with iso-contours of temperature are shown in Fig.7 along with the studied measurement locations. This section of interest corresponds to the refinement region in the two meshes. The main properties of the two meshes are listed in Tab.3. The coarse one is a rectangular domain, with a width of $1.4 \mathrm{~m}$ and a length of $2 \mathrm{~m}$. A fully tetrahedral unstructured mesh is applied in this case, an element size of $60 \mathrm{~mm}$ is set in the far field, the mesh around the jet is refined in 3 steps, with a mesh size of $4 \mathrm{~mm}$ up to $x / D=125,2 \mathrm{~mm}$ up to $x / D=45$, and $1 \mathrm{~mm}$ up to $x / D=22.5$. The refinement regions are following the expected jet spreading angle.

The fine mesh has a reduced domain size with a width of $0.24 \mathrm{~m}$ and a length of $0.48 \mathrm{~m}$. Up to $x / D=30$ the mesh is extruded from the plane of the inlet with a size of $0.5 \mathrm{~mm}$ in the axial direction. The remaining part $(x / D>30)$ is a buffer region with unstructured mesh. The inlet plane is meshed with a base size of $5 \mathrm{~mm}$, it has a refinement region with a $0.06 \mathrm{~m}$ 
radius with a size of $1 \mathrm{~mm}$, that decreases to $0.25 \mathrm{~mm}$ in the vicinity of the nozzle.

\begin{tabular}{l|ccc} 
& $\Delta_{\text {axial }}$ & $\Delta_{\text {radial }}$ & DoF \\
\hline Coarse & $1.0 \mathrm{~mm}$ & $1.0 \mathrm{~mm}$ & $970 \mathrm{k}$ \\
Fine & $0.5 \mathrm{~mm}$ & $0.2 . .1 .0 \mathrm{~mm}$ & $6.9 \mathrm{M}$
\end{tabular}

Table 3: DLR-A jet mesh size

(1)
the same surface mesh is applied for the fuel inlet in the two cases, so that the same turbulent boundary condition is imposed. The center of the inlet is meshed with a size of $0.2 \mathrm{~mm}$ and is refined near the edge of the inlet. The synthetic turbulent inlet strategy of [30] is adapted. In an a priori sensitivity study, we found the adequate turbulent length scale to be $3.5 \%$ of the diameter. A fully developed turbulent pipe flow profile is imposed for the mean and RMS values. The mean profile is reconstructed from the log-law using second order continuous polynomial interpolation in the core and buffer regions, while the RMS profiles are taken from the DNS data of [31].

The results of the present LES calculation with comparison of the measurement data of Meier et al. [29] are shown in Fig.8 and Fig.9 for analysis. The results show similar trends as those observed in the previous cases, but with more clear evidence of the influence of the discretization of the convective term. The results using the EMAC operator have shown improved predictions for fluctuating quantities, but also for mean values in this case. At upstream locations $(x / D=5)$ the axial velocity and mixture fraction are 

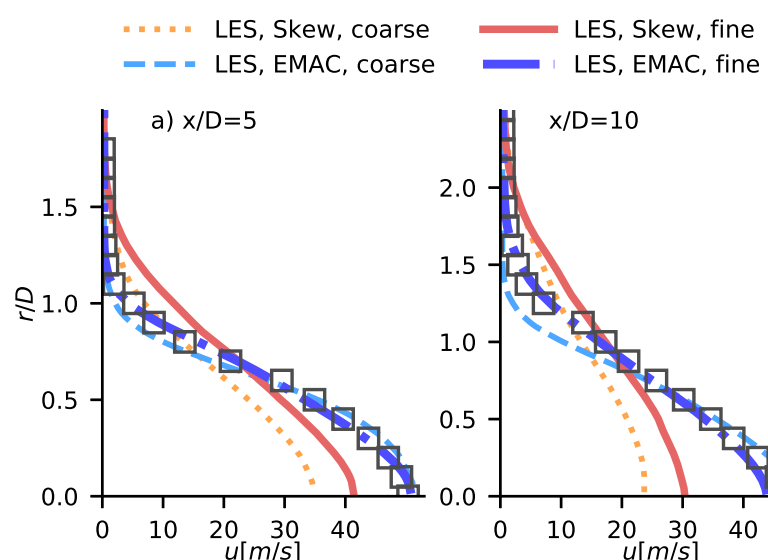

Meier et al. (2000)
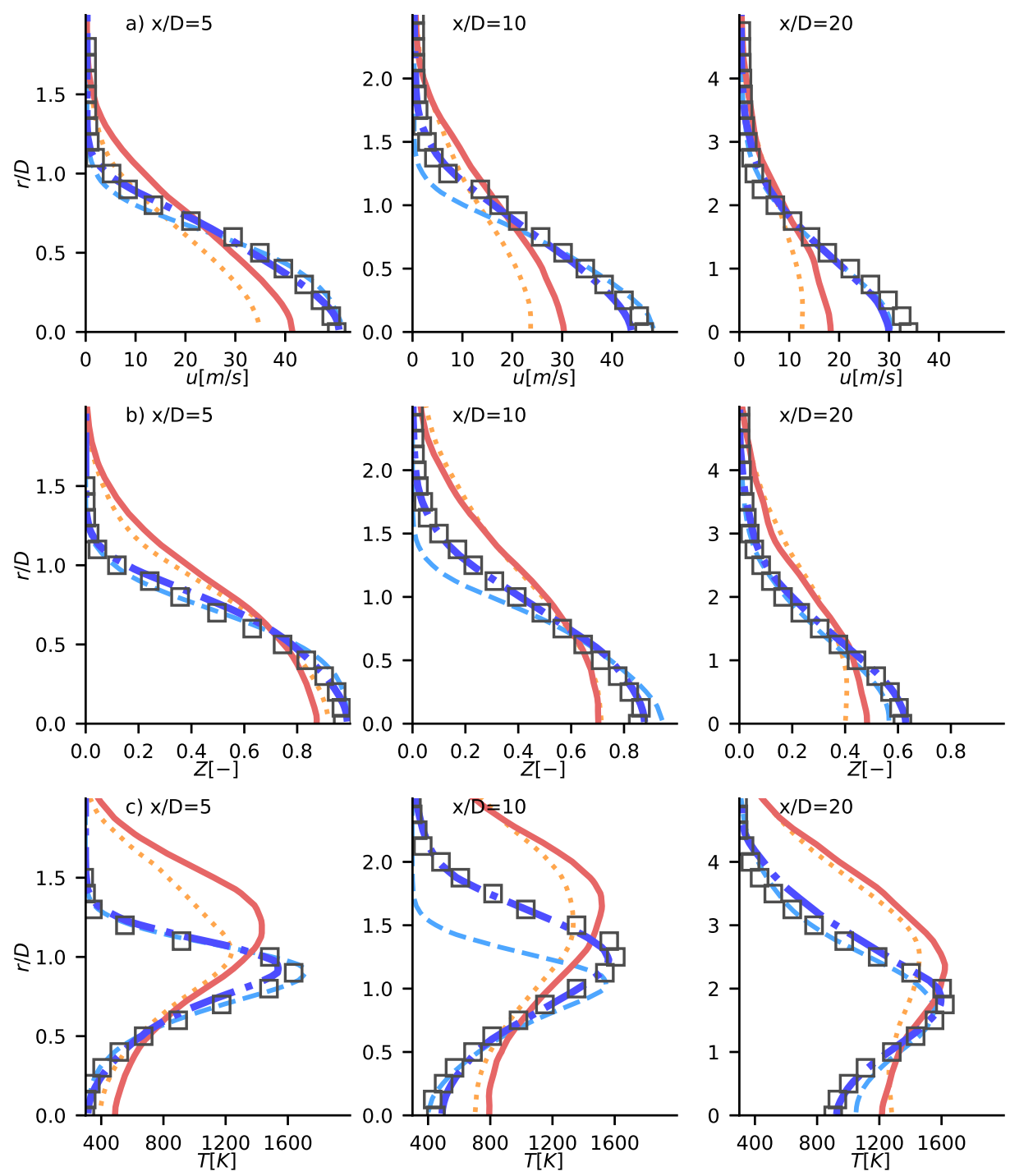

Figure 8: Comparison of mean profiles in the DLR A jet flame at three axial locations: $x \in$ $\{5 D, 10 D, 20 D\}$; a: mean axial velocity, b: mean mixture fraction, c: mean temperature; suqares: measurement of Meier et al.[29], dashed blue line: EMAC on coarse mesh, dotted orange line: skew-symmetric on coarse mesh, dash-dotted blue line: EMAC on fine mesh, solid red line: skew-symmetric on fine mesh 

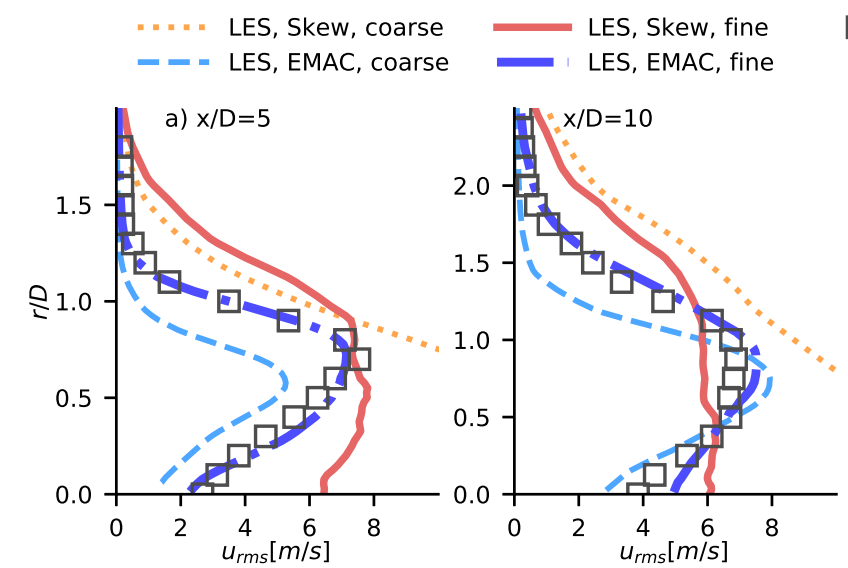

Meier et al. (2000)
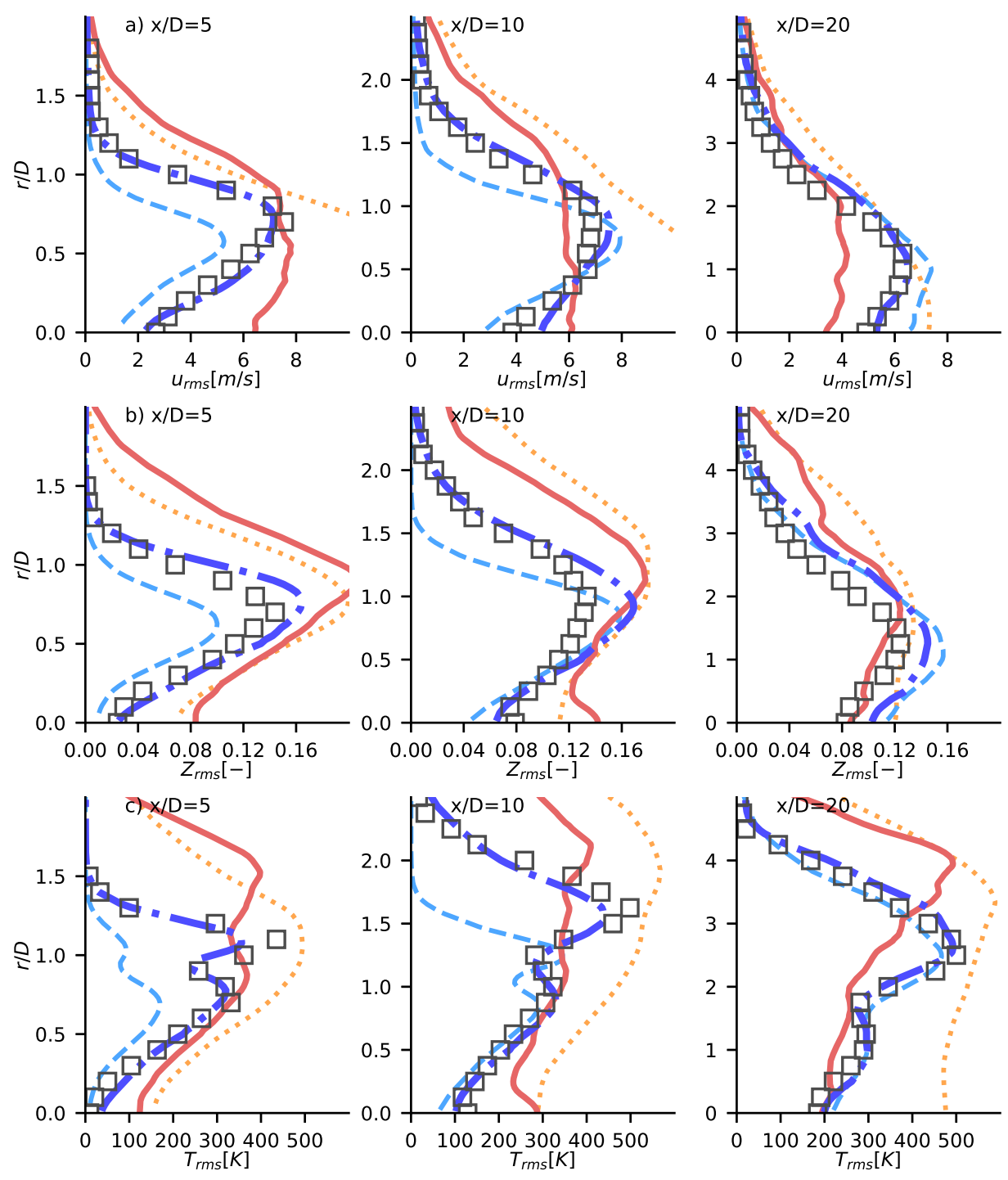

Figure 9: Comparison of RMS profiles in the DLR A jet flame at three axial locations: $x \in\{5 D, 10 D, 20 D\}$; a: axial velocity RMS, b: mixture fraction RMS, c: temperature RMS; squares: measurement of Meier et al.[29], dashed blue line: EMAC on coarse mesh, dotted orange line: skew-symmetric on coarse mesh, dash-dotted blue line: EMAC on fine mesh, solid red line: skew-symmetric on fine mesh 
in good agreement with experimental data. Jet penetration and spreading are well captured at this distance, which results in an accurate prediction of the temperature. The impact of the mesh resolution for EMAC at this axial location is quite low, though it becomes more relevant at downstream locations. In this test case, the EMAC discretization is clearly superior on both meshes. The mean profiles are predicted considerably well even on the coarse mesh except the edge of the jet at the $x / D=10$ axial location (Fig.8). The refinement corrects this discrepancy, and improves the RMS values (Fig.9). The skew-symmetric operator is found to be quite sensitive to the mesh resolution, but the results even with the fine mesh are still poor compared to those with EMAC, see Fig. 8 a,b. At more downstream locations, see Fig. 8 at $x / D=10$ and $x / D=20$, the same trend is maintained and the penetration of the jet is better predicted by EMAC. As the axial velocity exhibits a fast decay when using the skew-symmetric approximation, the mixture fraction deviates substantially from the reference values with flattened profiles. This results in large over-predictions of the flame temperature and jet spreading. The results using EMAC show an excellent correlation of velocity and mixture faction at all locations for mean and fluctuating quantities, and therefore predicts all flow parameters better.

Analysing the momentum conservation of the skew-symmetric operator in this case gives further insight on this issue. The jet shows self similar properties in the measurement for axial velocity and mixture fraction. These two properties are also similar to each other in the studied locations for the measurement and the EMAC operator (Fig.8 a,b). However the fluctuating heat release in the reacting layer surrounding the jet induces the momentum 
loss in the skew-symmetric case. The discrepancies in the mixture fraction profile are a consequence of the error in velocity, thus the similarity between the two quantities is broken.

\section{Conclusions}

In this study, we present a low-dissipation finite element scheme for low Mach number flows in the context of large-eddy simulation using an explicit third order Runge-Kutta method in conjunction with appropriate subgrid models.

The low Mach number approximation of the Navier-Stokes equations have been introduced in the weak form. We derived the energy, momentum, and angular momentum conserving formulation of the non-lineal term of the momentum equation for variable density flows. The symmetry properties of the original incompressible implementation are preserved by introducing a change of variables: $\mathbb{A} \equiv \sqrt{\rho} \mathbf{u}$. The conservation properties of the EMAC and the well-known skew-symmetric operator are derived for specific test conditions, where mass conservation is not strictly imposed. As postulated, by writing in terms of $\mathbb{A}$, the EMAC formulation preserves its conservative properties for variable density flows.

The complete spatio-temporal discretization scheme using finite elements and explicit time integration is presented. We exploit the stabilizing effect introduced by using an approximate Laplacian to stabilize the pressure equation. Otherwise no additional stabilization is introduced for the mass and momentum conservation. The error of kinetic energy conservation is of order $\mathcal{O}\left(\delta t h^{k+1}\right)$, thus dissipation is limited. 
The strategy has been tested on three physical problems: a fully developed anisothermal channel flow, a premixed swirling flame, and a nonpremixed jet flame. Both the skew-symmetric and the EMAC operators perform well in the anisothermal channel flow. The difference between the two strategies is minor. In general we observe higher discrepancies on the cold side, where the Reynolds number is higher, since a symmetric mesh is applied. Mesh refinement is shown to improve the results with both operators.

In the reacting cases, the EMAC discretization clearly performs better. It captures well both the velocity peaks and the central recirculation zone of the swirling flame. The skew-symmetric strategy produces high discrepancies in these locations, and it consistently underpredicts the fluctuations. In general it appears to give a more diffusive solution. In the DLR-A jet flame the level of complexity of the problem is increased. The results with the EMAC operator agree very well with the measurements, and mesh refinement leads to a clear improvement. On the other hand the error introduced by the skew-symmetric formulation breaks the relationship between the scalar and velocity fields of the jet, thus the error in the fluctuating properties is less deterministic.

Overall the proposed low-dissipation scheme with the EMAC discretization shows good agreement with the reference data in all test cases. The benefit of using the EMAC discretization with respect the classical skewsymmetric is more relevant in the reacting cases. We postulate this is caused by the lack of volumetric source term in anisothermal channel flow case. Specifically the heat release from combustion causes high density variations 
in a Lagrangian frame of reference, this effect is lacking in the channel case, where the density of a fluid particle is primarily modified by molecular and subgrid thermal diffusion. To conclude, in all three cases the performance of the proposed strategy is rather good and it has been capable of reproducing the results with excellent accuracy outperforming the classical skewsymmetric approach.

\section{Acknowledgements}

This project has received funding from the Spanish Ministry of Economy and Competitiveness in the frame of the CHEST project (TRA2017-89139C2-2-R) and from the European Union's Horizon 2020 research and innovation programme under the Marie Skłodowska-Curie grant agreement No. 713673. Ambrus Both has received financial support from "la Caixa" Fundation (ID 100010434). The fellowship grant code is LCF/BQ/IN17/11620032. Conflict of Interest: Daniel Mira acknowledges the Juan de la Cierva personal grant IJCI-2015-26686.

\section{References}

\section{References}

[1] T. Poinsot, D. Veynante, Theoretical and numerical combustion, RT Edwards, Inc., 2005.

[2] P. Moin, S. V. Apte, Large-eddy simulation of realistic gas turbine combustors, AIAA journal 44 (2006) 698-708. 
[3] D. Mira, M. Vázquez, G. Houzeaux, S. Gövert, J. W. Kok, E. Mahiques, L. Panek, Study of the wall thermal condition effect in a leanpremixed downscaled can combustor using large-eddy simulation, in: ASME. Turbo Expo: Power for Land, Sea, and Air, Volume 5B: Heat Transfer, GT2016-58016, American Society of Mechanical Engineers, p. V05BT17A018.

[4] H. Pitsch, Large-eddy simulation of turbulent combustion, Annu. Rev. Fluid Mech. 38 (2006) 453-482.

[5] S. Charnyi, T. Heister, M. A. Olshanskii, L. G. Rebholz, On conservation laws of navier-stokes galerkin discretizations, Journal of Computational Physics 337 (2017) 289-308.

[6] R. Verstappen, A. Veldman, Symmetry-preserving discretization of turbulent flow, Journal of Computational Physics 187 (2003) 343-368.

[7] J. Reiss, A family of energy stable, skew-symmetric finite difference schemes on collocated grids, Journal of Scientific Computing 65 (2015) 821-838.

[8] F. Nicoud, Conservative high-order finite-difference schemes for lowmach number flows, Journal of Computational Physics 158 (2000) 7197.

[9] Y. Morinishi, Skew-symmetric form of convective terms and fully conservative finite difference schemes for variable density low-mach number flows, Journal of Computational Physics 229 (2010) 276-300. 
[10] J. Brouwer, J. Reiss, J. Sesterhenn, Conservative time integrators of arbitrary order for finite-difference discretization of compressible flow, Computers \& Fluids 100 (2014) 1-12.

[11] W. Rozema, R. W. Verstappen, A. E. Veldman, J. C. Kok, Lowdissipation simulation methods and models for turbulent subsonic flow, Archives of Computational Methods in Engineering (2018) 1-32.

[12] G. Coppola, F. Capuano, S. Pirozzoli, L. de Luca, Numerically stable formulations of convective terms for turbulent compressible flows, Journal of Computational Physics 382 (2019) 86-104.

[13] R. Codina, J. Principe, M. Ávila, Finite element approximation of turbulent thermally coupled incompressible flows with numerical sub-grid scale modelling, International Journal of Numerical Methods for Heat \& Fluid Flow 20 (2010) 492-516.

[14] O. Lehmkuhl, G. Houzeaux, H. Owen, G. Chrysokentis, I. Rodriguez, A low-dissipation finite element scheme for scale resolving simulations of turbulent flows, Journal of Computational Physics 390 (2019) 51-65.

[15] E. R. Van Driest, Turbulent boundary layer in compressible fluids, Journal of Aeronautical Sciences 18 (1951) 145-160.

[16] C.-S. Yih, A transformation for non-homentropic flows, with an application to large-amplitude motion in the atmosphere, Journal of Fluid Mechanics 9 (1960) 68-80.

[17] S. Kida, S. A. Orszag, Energy and spectral dynamics in decaying compressible turbulence, Journal of Scientific Computing 7 (1992) 1-34. 
[18] A. W. Cook, Y. Zhou, Energy transfer in rayleigh-taylor instability, Physical Review E 66 (2002) 026312.

[19] Y. V. Peet, S. K. Lele, Computational framework for coupling compressible and low mach number codes, AIAA journal 46 (2008) 1990-2001.

[20] F. Capuano, G. Coppola, M. Chiatto, L. de Luca, Approximate projection method for the incompressible navier-stokes equations, AIAA Journal 54 (2016) 2179-2182.

[21] R. Codina, J. Blasco, A finite element formulation for the stokes problem allowing equal velocity-pressure interpolation, Computer Methods in Applied Mechanics and Engineering 143 (1997) 373-391.

[22] F. Trias, O. Lehmkuhl, A self-adaptive strategy for the time integration of navier-stokes equations, Numerical Heat Transfer, Part B: Fundamentals 60 (2011) 116-134.

[23] A. Toutant, F. Bataille, Turbulence statistics in a fully developed channel flow submitted to a high temperature gradient, International Journal of Thermal Sciences 74 (2013) 104-118.

[24] A. Vreman, An eddy-viscosity subgrid-scale model for turbulent shear flow: Algebraic theory and applications, Physics of fluids 16 (2004) $3670-3681$.

[25] W. Meier, P. Weigand, X. Duan, R. Giezendanner-Thoben, Detailed characterization of the dynamics of thermoacoustic pulsations in a lean premixed swirl flame, Combustion and Flame 150 (2007) 2-26. 
[26] S. Gövert, D. Mira, J. B. Kok, M. Vázquez, G. Houzeaux, The effect of partial premixing and heat loss on the reacting flow field prediction of a swirl stabilized gas turbine model combustor, Flow, turbulence and combustion 100 (2018) 503-534.

[27] G. P. Smith, D. M. Golden, M. Frenklach, N. W. Moriarty, B. Eiteneer, M. Goldenberg, C. T. Bowman, R. K. Hanson, S. Song, W. Gardiner Jr, et al., Gri-mech 3.0, 1999, URL http://www. me. berkeley. edu/gri_mech (2011).

[28] S. Gövert, D. Mira, J. B. Kok, M. Vázquez, G. Houzeaux, Turbulent combustion modelling of a confined premixed jet flame including heat loss effects using tabulated chemistry, Applied energy 156 (2015) 804815.

[29] W. Meier, R. Barlow, Y.-L. Chen, J.-Y. Chen, Raman/rayleigh/lif measurements in a turbulent ch $4 / \mathrm{h} 2 / \mathrm{n} 2$ jet diffusion flame: experimental techniques and turbulence-chemistry interaction, Combustion and Flame 123 (2000) 326-343.

[30] A. Kempf, M. Klein, J. Janicka, Efficient generation of initial-and inflowconditions for transient turbulent flows in arbitrary geometries, Flow, Turbulence and combustion 74 (2005) 67-84.

[31] G. K. El Khoury, P. Schlatter, A. Noorani, P. F. Fischer, G. Brethouwer, A. V. Johansson, Direct numerical simulation of turbulent pipe flow at moderately high reynolds numbers, Flow, turbulence and combustion 91 (2013) 475-495. 